\title{
SYMMETRY DIFFEOMORPHISM GROUP OF A MANIFOLD OF NONPOSITIVE CURVATURE
}

\author{
PATRICK EBERLEIN
}

\begin{abstract}
Let $\tilde{M}$ denote a complete simply connected manifold of nonpositive sectional curvature. For each point $p \in \tilde{M}$ let $s_{p}$ denote the diffeomorphism of $\tilde{M}$ that fixes $p$ and reverses all geodesics through $p$. The symmetry diffeomorphism group $G^{*}$ generated by all diffeomorphisms $\left\{s_{p}: p \in \tilde{M}\right\}$ extends naturally to group of homeomorphisms of the boundary sphere $\tilde{M}(\infty)$. A subset $X$ of $\tilde{M}(\infty)$ is called involutive if it is invariant under $G^{*}$.
\end{abstract}

THEOREM. Let $X \subseteq \tilde{M}(\infty)$ be a proper, closed involutive subset. For each point $p \in \tilde{M}$ let $N(p)$ denote the linear span in $T_{p} \tilde{M}$ of those vectors at $p$ that are tangent to a geodesic $\gamma$ whose asymptotic equivalence class $\gamma(\infty)$ belongs to $X$. If $N(p)$ is a proper subspace of $T_{p} \tilde{M}$ for some point $p \in \tilde{M}$, then $\tilde{M}$ splits as a Riemannian product $\tilde{M}_{1} \times \tilde{M}_{2}$ such that $N$ is the distribution of $\tilde{M}$ induced by $\tilde{M}_{1}$.

This result has several applications that include new results as well as great simplifications in the proofs of some known results. In a sequel to this paper it is shown that if $\tilde{M}$ is irreducible and $\tilde{M}(\infty)$ admits a proper, closed involutive subset $X$, then $\tilde{M}$ is isometric to a symmetric space of noncompact type and rank $k \geq 2$.

Introduction. Let $\tilde{M}$ denote a complete, simply connected manifold of nonpositive sectional curvature. It is well known that if $p, q$ are any two distinct points of $\tilde{M}$, then there is a unique geodesic $\gamma_{p q}$ that joins $p$ to $q$. Hence for each point $p$ of $\tilde{M}$ we obtain a symmetry diffeomorphism $s_{p}: \tilde{M} \rightarrow \tilde{M}$ given by $s_{p} \gamma(t)=\gamma(-t)$ for all geodesics $\gamma$ of $\tilde{M}$ with $\gamma(0)=p$ and for all $t \in \mathbf{R}$. We define the symmetry diffeomorphism group $G^{*}$ to be the group of diffeomorphisms of $\tilde{M}$ generated by the geodesic symmetries $s_{p}, p \in \tilde{M}$. The manifold $\tilde{M}$ is said to be a symmetric space if $G^{*} \subseteq I(\tilde{M})$, the isometry group of $\tilde{M}$, and $\tilde{M}$ is said to be symmetric of noncompact type if $\tilde{M}$ is a symmetric space and has a trivial Euclidean de Rham factor.

If $\tilde{M}(\infty)$ denotes the boundary sphere of an arbitrary manifold $\tilde{M}$ (see $\S 1$ for definitions and details), then the group $G^{*}$ acts on $\tilde{M}(\infty)$ by homeomorphisms in a natural way. A subset $X \subseteq \tilde{M}(\infty)$ is said to be involutive if $X$ is invariant under the group $G^{*}$. In this paper and its sequel [E8] we investigate the consequences when $\tilde{M}(\infty)$ admits a proper involutive subset $X$ that is closed in the sphere topology of $\tilde{M}(\infty)$. The results fall into two categories: splitting theorems for $\tilde{M}$ and characterizations of symmetric spaces of noncompact type and rank at least two.

Received by the editors June $30,1987$.

1980 Mathematics Subject Classification (1985 Revision). Primary 53C20; Secondary 53C35.

Supported in part by NSF Grants MCS-8219609 and DMS-8601367. 
Note that a priori the group $G^{*}$ need not contain any isometries of $\tilde{M}$ other than the identity.

Let $X \subseteq \tilde{M}(\infty)$ be a proper, closed involutive subset. For each point $p$ of $\tilde{M}$ let $\mathscr{N}(p)$ denote the linear span in $T_{p} \tilde{M}$ of $\{V(p, x): x \in X\}$, where $V(p, x)$ denotes the initial velocity of the unique geodesic $\gamma_{p x}$ that starts at $p$ and belongs to $x$ (compare $\S 1$ ). The main result of this paper, Theorem $\mathrm{A}$ of $\S 3$, is that if $\mathscr{N}(p)$ is a proper subspace of $T_{p} \tilde{M}$ for some point $p$ of $\tilde{M}$, then $\tilde{M}$ splits as a nontrivial Riemannian product $\tilde{M}_{1} \times \tilde{M}_{2}$ such that the foliation of $\tilde{M}$ induced by $\tilde{M}_{1}$ is precisely the distribution $\mathscr{N}$. This result leads to simplified proofs of many known splitting theorems (see $\S \S 5$ and 6), and in addition gives a cohesiveness to these theorems by putting them in a unified context.

The idea of an involutive subset $X \subseteq \tilde{M}(\infty)$ plays an important role in the proof of the main result of [E6], which is a special case of the Gromov Rigidity Theorem [BGS, $\S \S 14$ and 15]; see especially Proposition 4.3 of [E6]. The symmetry diffeomorphism group $G^{*}$ acting on $\tilde{M}(\infty)$ also plays a role in [BS]. The statement of Theorem A has its origin in the discussion of $\S 5$ of [E6], but the actual proof of Theorem A is very similar to the proof of a splitting theorem from [BGS, p. 222], which in fact is a special case of Theorem A.

In the sequel [E8] to this paper we show that if $\tilde{M}$ is irreducible in the sense of de Rham and admits a proper, closed involutive subset $X \subseteq \tilde{M}(\infty)$, then $\tilde{M}$ is isometric to a symmetric space of noncompact type and rank $k \geq 2$. This result yields as corollaries several characterizations of symmetric spaces of noncompact type and rank at least two, and in particular leads to simplified proofs of the Gromov Rigidity Theorem and the Rank Rigidity Theorem of Ballmann [B] and Burns-Spatzier [BS].

1. Preliminaries. In this section we summarize briefly some facts that will be useful later. For general references see [BO, EO and BBE].

(1.1) Notation. $\tilde{M}$ will always denote a complete, simply connected Riemannian manifold with nonpositive sectional curvature. All geodesics of $\tilde{M}$ will be assumed to have unit speed. $I(\tilde{M})$ denotes the group of isometries of $\tilde{M}$, and $I_{0}(\tilde{M})$ denotes the connected component of $I(\tilde{M})$ that contains the identity. $S \tilde{M}$ denotes the unit tangent bundle of $\tilde{M}$. Given $v \in S \tilde{M}$, the geodesic with initial velocity $v$ is denoted by $\gamma_{v}$.

Geodesics $\gamma, \sigma$ of $\tilde{M}$ are asymptotic if $d(\gamma t, \sigma t) \leq c$ for all $t \geq 0$ and some $c>0$. $\tilde{M}(\infty)$ will denote the set of equivalence classes of asymptotic geodesics of $\tilde{M}$. Equipped with a natural sphere (cone) topology $\tilde{M}(\infty)$ is homeomorphic to an $(n-1)$ sphere, where $n=\operatorname{dim} \tilde{M}$. For a geodesic $\gamma$ of $\tilde{M}$ we let $\gamma(\infty), \gamma(-\infty)$ denote the asymptotic equivalence classes of $\gamma$ and $\gamma^{-1}: t \rightarrow \gamma(-t)$. Given points $p \in \tilde{M}$ and $x \in \tilde{M}(\infty)$ there exists a unique geodesic $\gamma_{p x}$ such that $\gamma_{p x}(0)=p$ and $\gamma_{p x}(\infty)=x . V(p, x)$ will denote the unit vector $\gamma_{p x}^{\prime}(0)$. The angle subtended at a point $p$ in $\tilde{M}$ by points $x, y$ in $\tilde{M}(\infty)$ is denoted by $\measuredangle_{p}(x, y)$ and is defined to be the angle between $V(p, x)$ and $V(p, y)$.

(1.2) Busemann functions and horospheres. Given a point $x \in \tilde{M}(\infty)$ and a point $p \in \tilde{M}$ one defines a Busemann function $f_{x}$ at $x$ by

$$
f_{x}(q)=\lim _{t \rightarrow+\infty} d\left(q, \gamma_{p x} t\right)-t
$$


For different points $p_{1}, p_{2}$ in $\tilde{M}$ the corresponding Busemann functions at a fixed point $x$ in $\tilde{M}(\infty)$ differ in $\tilde{M}$ by a constant. For any $x \in \tilde{M}(\infty)$ a Busemann function $f_{x}$ at $x$ is $C^{2}$ and convex, and $\operatorname{grad} f_{x}(p)=-V(p, x)$. Hence $\mid f_{x}(p)-$ $f_{x}(q) \mid \leq d(p, q)$ for any $x \in \tilde{M}(\infty)$ and any points $p, q \in \tilde{M}$.

If $f_{x}$ is a Busemann function at $x \in \tilde{M}(\infty)$ and if $p \in \tilde{M}$ is any point, then one defines $H(p, x)=\left\{q \in \tilde{M}: f_{x}(q)=f_{x}(p)\right\}$ and $B(p, x)=\left\{q \in \tilde{M}: f_{x}(q) \leq f_{x}(p)\right\}$. The sets $H(p, x)$ and $B(p, x)$ are called the horosphere and horoball through $p$ determined by $x$. The set $B(p, x)$ is a proper closed convex subset of $\tilde{M}$ by the convexity of $f_{x}$.

(1.3) Riemannian splittings of $\tilde{M}$. Let $\tilde{M}=\tilde{M}_{1} \times \tilde{M}_{2}$ be a Riemannian product manifold. This splitting of $\tilde{M}$ is said to be invariant under a group $\Gamma \subseteq I(\tilde{M})$ if for each $\varphi \in \Gamma$ and each $p \in \tilde{M}$ we have $d \varphi\left[\mathscr{N}_{i}(p)\right]=\mathscr{N}_{i}(\varphi p)$ for $i=1,2$, where $\mathscr{N}_{i}$ is the foliation of $\tilde{M}$ induced by $\tilde{M}_{i}$. If a group $\Gamma \subseteq I(\tilde{M})$ leaves invariant a splitting $\tilde{M}=\tilde{M}_{1} \times \tilde{M}_{2}$, then it follows that every element $\varphi$ of $\Gamma$ can be written $\varphi=\left(\varphi_{1}, \varphi_{2}\right)$, where $\varphi_{i} \in I\left(\tilde{M}_{i}\right)$ for $i=1,2$. In this case one has projection homomorphisms $p_{i}: \Gamma \rightarrow I\left(\tilde{M}_{i}\right)$ given by $p_{i}(\varphi)=\varphi_{i}$ for $i=1,2$. If $\tilde{M}=\tilde{M}_{1} \times \tilde{M}_{2}$ is a Riemannian product manifold with a trivial Euclidean de Rham factor, then every group $\Gamma \subseteq I(\tilde{M})$ admits a finite index subgroup $\Gamma^{*}$ that leaves the splitting invariant.

(1.4) Clifford translations. For any isometry $\varphi$ of $\tilde{M}$ one defines the displacement function $d_{\varphi}: \tilde{M} \rightarrow \mathbf{R}$ given by $d_{\varphi}(p)=d(p, \varphi p)$. The function $d_{\varphi}$ is convex and its minimum locus, if nonempty, is the union of all geodesics in $\tilde{M}$ that are translated by $\varphi$.

If $d_{\varphi}$ is constant in $\tilde{M}$, then the isometry $\varphi$ is called a Clifford translation of $\tilde{M}$. If $d_{\varphi}$ is bounded above in $\tilde{M}$, then $\varphi$ is a Clifford translation of $\tilde{M}$ by elementary convexity properties. Let $C(\tilde{M})$ denote the subgroup of $I(\tilde{M})$ consisting of all Clifford translations.

Write $\tilde{M}=\tilde{M}_{0} \times \tilde{M}_{1}$, where $\tilde{M}_{0}$ is the Euclidean de Rham factor of $\tilde{M}$ and $\tilde{M}_{1}$ is the product of all irreducible non-Euclidean de Rham factors of $\tilde{M}$. A result of [W1] states that $C(\tilde{M})=\left\{\varphi \in I(\tilde{M}): \varphi=\left(\varphi_{0}\right.\right.$,id), where $\varphi_{0}$ is a translation of $\left.\tilde{M}_{0}\right\}$. In particular $C(\tilde{M})$ is a normal abelian subgroup of $I(\tilde{M})$.

(1.5) Limit sets. If $\Gamma \subseteq I(\tilde{M})$ is any subgroup and $p \in \tilde{M}$ is any point, then one defines the limit set $L(\Gamma)$ to be the set of accumulation points in $\tilde{M}(\infty)$ of the orbit $\Gamma(p)$. The set $L(\Gamma)$ is a closed subset of $\tilde{M}(\infty)$ in the sphere topology. $L(\Gamma)$ does not depend on the point $p \in \tilde{M}$, and hence $L(\Gamma)$ is invariant under all isometries of $\tilde{M}$ that normalize $\Gamma$.

For later use we need the following.

Proposition. Let $\Gamma \subseteq I(\tilde{M})$ be a subgroup such that $L(\Gamma)=\tilde{M}(\infty)$, and let $N$ be a nonidentity normal subgroup of $\Gamma$. Then $L(N)$ is nonempty.

ProOF. Let $N, \Gamma$ be as in the statement of the proposition. If $L(N)$ is empty, then the orbit $N(p)$ is bounded in $\tilde{M}$ for every $p \in \tilde{M}$ and hence $N$ has a fixed point in $\tilde{M}$ by the Cartan fixed point theorem. The set of points in $\tilde{M}$ fixed by $N$ is closed, convex and invariant under $\Gamma$. Since $L(\Gamma)=\tilde{M}(\infty)$ it follows that $N$ fixes every point of $\tilde{M}$, which contradicts the hypothesis that $N \neq\{1\}$. 
(1.6) Duality. Let $\Gamma \subseteq I(\tilde{M})$ be any group. Two points $x, y$ in $\tilde{M}(\infty)$, not necessarily distinct, are said to be $\Gamma$-dual if there exists a sequence $\left\{\varphi_{n}\right\} \subseteq \Gamma$ such that $\varphi_{n}(p) \rightarrow x$ and $\varphi_{n}^{-1}(p) \rightarrow y$ as $n \rightarrow \infty$ for any point $p$ of $\tilde{M}$. It is routine to verify that the set of points in $\tilde{M}(\infty)$ that are $\Gamma$-dual to a given point $x \in \tilde{M}(\infty)$ is closed in $\tilde{M}(\infty)$ and invariant under $\Gamma$. The next result, whose proof is contained in the proof of Lemma 2.4a of [CE], will be useful later.

Proposition 1.6a. Let $1 \neq \Gamma \subseteq I(\tilde{M})$ be any group, and let $x, z$ be points in $\tilde{M}(\infty)$ that are $\Gamma$-dual. If $p \in \tilde{M}$ is arbitrary and $y=\gamma_{p x}(-\infty) \in \tilde{M}(\infty)$, then $z \in \overline{\Gamma(y)}$.

A group $\Gamma \subseteq I(\tilde{M})$ is said to satisfy the duality condition if $\gamma(\infty)$ and $\gamma(-\infty)$ are $\Gamma$-dual for any geodesic $\gamma$ of $\tilde{M}$. If $\Gamma \subseteq I(\tilde{M})$ is a discrete group such that the quotient space $\tilde{M} / \Gamma$ is a smooth manifold of finite Riemannian volume, then $\Gamma$ satisfies the duality condition by Proposition 3.7 of [E2] and the Poincaré recurrence lemma.

Proposition 1.6b. Let $\Gamma \subseteq I(\tilde{M})$ satisfy the duality condition. Then $\Gamma$ has the following properties:

(1) If $\Gamma^{*}$ is a subgroup of $\Gamma$ with finite index, then $\Gamma^{*}$ satisfies the duality condition.

(2) If $\Gamma$ leaves invariant a Riemannian splitting $\tilde{M}=\tilde{M}_{1} \times \tilde{M}_{2}$, then $p_{i}(\Gamma)$ satisfies the duality condition in $\tilde{M}_{i}$ for $i=1,2$, where $p_{i}: \Gamma \rightarrow I\left(\tilde{M}_{i}\right)$ denotes the projection homomorphism.

(3) If $x \in \tilde{M}(\infty)$ is arbitrary, then $\overline{\Gamma(y)}=\overline{\Gamma(x)}$ for all $y \in \overline{\Gamma(x)}$.

Proof. Property (1) is proved in Appendix I of [E3] while property (3) is proved in Lemma 2.9 of [BBE]. Property (2) follows from the definition of the duality condition and geometric properties of Riemannian product manifolds.

2. The symmetry diffeomorphism group. Let $\tilde{M}$ be an arbitrary complete, simply connected Riemannian manifold with nonpositive sectional curvature. For each point $p$ in $\tilde{M}$ one defines the geodesic symmetry $s_{p}: \tilde{M} \rightarrow \tilde{M}$ given by

$$
s_{p}(\gamma(t))=\gamma(-t)
$$

for all geodesics $\gamma$ of $\tilde{M}$ with $\gamma(0)=p$ and for all $t \in \mathbf{R}$. The map $s_{p}$ fixes $p$ and is a diffeomorphism of $\tilde{M}$. We define

$$
\begin{aligned}
G^{*}= & \text { the subgroup of diffeomorphisms of } \tilde{M} \\
& \text { generated by the geodesic symmetries }\left\{s_{p}: p \in \tilde{M}\right\} .
\end{aligned}
$$

$$
\begin{aligned}
G_{e}^{*}= & \text { the subgroup of } G^{*} \text { consisting of } \\
& \text { elements that are the product of an even } \\
& \text { number of geodesic symmetries. }
\end{aligned}
$$

The group $G^{*}$ is called the symmetry diffeomorphism group of $\tilde{M}$. The subgroup $G_{e}^{*}$, which has index 2 and hence is normal in $G^{*}$, is called the group of even symmetry diffeomorphisms (compare [L, p. 64]). 
(2.3) $G^{*}$ acting on $\tilde{M}(\infty)$. For each point $p \in \tilde{M}$ we define $s_{p}: \tilde{M}(\infty) \rightarrow \tilde{M}(\infty)$ by

$$
s_{p}(x)=\gamma_{p x}(-\infty)
$$

for every $x \in \tilde{M}(\infty)$. The group $G^{*}$ now acts on $\tilde{M}(\infty)$ by homeomorphisms. Note that $\varphi \circ s_{p} \circ \varphi^{-1}=s_{\varphi(p)}$ for all $\varphi \in I(\tilde{M})$ and all $p \in \tilde{M}$ so that $I(\tilde{M})$ normalizes both $G^{*}$ and $G_{e}^{*}$, regarded as groups acting on $\tilde{M}$ by diffeomorphisms or on $\tilde{M}(\infty)$ by homeomorphisms.

(2.4) Involutive subsets of $\tilde{M}(\infty)$.

Definition. A subset $X \subseteq \tilde{M}(\infty)$ is called involutive if $X$ is invariant under the symmetry diffeomorphism group $G^{*}$.

REMARKS. (a) If $X \subseteq \tilde{M}(\infty)$ is involutive, then the closure of $X$ in $\tilde{M}(\infty)$ is also involutive.

(b) If $X \subseteq \tilde{M}(\infty)$ is invariant under $G_{e}^{*}$, the subgroup of even symmetry diffeomorphisms, then $X \cup s_{p}(X)$ and $X \cap s_{p}(X)$ are involutive subsets for every point $p$ in $\tilde{M}$. This follows from the fact that $G_{e}^{*}$ is a normal subgroup of $G^{*}$ and hence $s_{p}(X)$ is $G_{e}^{*}$-invariant for every $p \in \tilde{M}$.

Examples of involutive subsets. (a) Let $\tilde{M}=\tilde{M}_{1} \times \tilde{M}_{2}$ be a Riemannian product manifold. Given any numbers $\alpha, \beta$ with $0 \leq \alpha, \beta \leq 1$ and $\alpha^{2}+\beta^{2}=1$ we define

$$
\begin{aligned}
\tilde{M}_{\alpha \beta}(\infty)=\{\gamma(\infty): \gamma(t) & =\left(\gamma_{1}(\alpha t), \gamma_{2}(\beta t)\right), \\
& \text { where } \left.\gamma_{i} \text { is a unit speed geodesic in } M_{i} \text { for } i=1,2\right\} .
\end{aligned}
$$

The set $\tilde{M}_{\alpha \beta}(\infty)$ is closed and involutive for each choice of $\alpha, \beta$. Note that $\tilde{M}_{\alpha \beta}(\infty)$ $=\tilde{M}_{1}(\infty)$ if $\alpha=1$ and $\beta=0$ and equals $\tilde{M}_{2}(\infty)$ if $\alpha=0$ and $\beta=1$.

(b) Let $\tilde{M}$ be a symmetric space of noncompact type. If $X \subseteq \tilde{M}(\infty)$ is invariant under the isometry group $I(\tilde{M})$, then $X$ is involutive since $G^{*} \subseteq I(\tilde{M})$. If $\tilde{M}$ has rank $k \geq 2$, then the closure in $\tilde{M}(\infty)$ of any orbit of $I(\tilde{M})$ is a proper subset of $\tilde{M}(\infty)$. Moreover, the set $X \subseteq \tilde{M}(\infty)$ consisting of singular points (compare $\S 3$ of [E6]) is proper, closed and invariant under $I(\tilde{M})$.

(2.5) Existence criteria for involutive subsets.

Proposition 2.5a. Let $\Gamma \subseteq I(\tilde{M})$ satisfy the duality condition, and let $X \subseteq$ $\tilde{M}(\infty)$ be closed and $\Gamma$-invariant. Then $X \cup s_{p}(X)$ and $X \cap s_{p}(X)$ are closed involutive subsets of $\tilde{M}(\infty)$ for any point $p$ of $\tilde{M}$.

PrOof. This is an immediate consequence of (b) in (3.2) above and the following.

LEMMA 2.5a. Let $\Gamma \subseteq I(\tilde{M})$ satisfy the duality condition, and let $X \subseteq \tilde{M}(\infty)$ be closed and $\Gamma$-invariant. Then $X$ is $G_{e}^{*}$-invariant.

PROOF OF THE LEMMA. It suffices to show that $s_{p} s_{q}(x) \in \overline{\Gamma(x)}$ for any points $p, q$ in $\tilde{M}$ and $x \in X$. If $y=s_{q}(x)$ and $x^{*}=s_{p}(y)=s_{p} s_{q}(x)$, then $y$ is $\Gamma$-dual to $x^{*}$ and the assertion of the lemma now follows from Proposition 1.6a.

COROLLARY 2.5a. Let $\Gamma \subseteq I(\tilde{M})$ satisfy the duality condition. If $\tilde{M}(\infty)$ admits a proper closed $\Gamma$-invariant subset, then $\tilde{M}(\infty)$ admits a proper closed involutive subset. 
Proof. Let $X \subseteq \tilde{M}(\infty)$ be a proper closed $\Gamma$-invariant subset. The sets $X \cup s_{p}(X)$ and $X \cap s_{p}(X)$ are closed and involutive for any point $p$ of $\tilde{M}$ by the proposition. If $X \cup s_{p}(X)=\tilde{M}(\infty)$ for some point $p$ of $\tilde{M}$, then $X \cap s_{p}(X)$ is nonempty since $\tilde{M}(\infty)$ is connected.

Proposition 2.5b. Let $\Gamma \subseteq \tilde{M}(\infty)$ satisfy the duality condition, and let $X \subseteq$ $\tilde{M}(\infty)$ be closed and $\Gamma$-invariant. Assume moreover that for each point $x \in X$ there exists $y \in X$ that is $\Gamma$-dual to $x$. Then $X$ is involutive.

Proof. Given $x \in X$ and $p \in \tilde{M}$ let $y=s_{p}(x) \in \tilde{M}(\infty)$. If $y^{*} \in X$ is $\Gamma$-dual to $x$, then by Proposition 1.6a we have $y^{*} \in \overline{\Gamma(y)}$, and hence $y \in \overline{\Gamma\left(y^{*}\right)} \subseteq X$ by (3) of Proposition 1.6b.

COROLLARY 2.5b. Let $\Gamma \subseteq I(\tilde{M})$ satisfy the duality condition, and let $1 \neq \Gamma_{0}$ be a normal subgroup of $\Gamma$. Then $L\left(\Gamma_{0}\right)$, the limit set of $\Gamma_{0}$, is a closed, nonempty involutive subset of $\tilde{M}(\infty)$.

PROOF. By the definition of $L\left(\Gamma_{0}\right)$, for every point $x \in L\left(\Gamma_{0}\right)$ there exists a point $y \in L\left(\Gamma_{0}\right)$ that is $\Gamma_{0}$-dual and hence $\Gamma$-dual to $x$. The assertion now follows from Proposition 2.5b and the proposition in (1.5).

Proposition 2.5c. Let $\Gamma \subseteq I(\tilde{M})$ be a subgroup with $L(\Gamma)=\tilde{M}(\infty)$. Let $X \subseteq \tilde{M}(\infty)$ be a closed $\Gamma$-invariant subset such that for each point $x \in X$ there exists $p \in \tilde{M}$ such that $s_{p}(x) \in X$. Then $X$ is involutive.

Proof. Given $x \in X$ and $q \in \tilde{M}$ let $y=s_{q}(x) \in \tilde{M}(\infty)$. Choose $\left\{\varphi_{n}\right\} \subseteq \Gamma$ so that $\varphi_{n}(q) \rightarrow y$ as $n \rightarrow \infty$ and let $\varphi_{n}^{-1}(q)$ converge to some point $z \in \tilde{M}(\infty)$ by passing to a subsequence if necessary. By Proposition 1.6a we see that $z \in \overline{\Gamma(x)} \subseteq$ $X$, and by hypothesis there exists a point $p \in \tilde{M}$ such that $w=s_{p}(z) \in X$. Since $y$ and $z$ are $\Gamma$-dual it follows by Proposition 1.6a that $y \in \overline{\Gamma(w)} \subseteq X$.

COROLlARY 2.5c. Let $B \subseteq \tilde{M}$ be a complete, totally geodesic submanifold, and let $X=B(\infty) \subseteq \tilde{M}(\infty)$. Let $\Gamma_{X}=\{\varphi \in I(\tilde{M}): \varphi(X) \subseteq X\}$. If $L\left(\Gamma_{X}\right)=\tilde{M}(\infty)$, then $X$ is involutive.

From the corollary above and Theorem $\mathrm{A}$ of the next section we obtain an alternate proof of Proposition 2.2 of [E3]. However, the main results in this paper, Theorem A and its applications, are related to Corollary $2.5 \mathrm{~b}$.

3. A splitting theorem. In this section we prove the following result:

THEOREM A. Let $\tilde{M}$ be an arbitrary complete, simply connected manifold of nonpositive sectional curvature, and let $X \subseteq \tilde{M}(\infty)$ be a closed involutive subset. For each point $p$ in $\tilde{M}$ define

$$
\begin{aligned}
B_{p} & =\text { closed convex hull of }\left\{\gamma_{p x}(\mathbf{R}): x \in X\right\}, \\
\mathscr{N}(p) & =\text { subspace of } T_{p} \tilde{M} \text { spanned by }\left\{\gamma_{p x}^{\prime}(0): x \in X\right\} .
\end{aligned}
$$

Then

(1) $B_{p}$ is a proper subset of $\tilde{M}$ for all points $p \in \tilde{M}$ if and only if $\mathcal{N}(p)$ is a proper subspace of $T_{p} \tilde{M}$ for some point $p \in \tilde{M}$. 
(2) If $\mathcal{N}(p)$ is a proper subspace of $T_{p} \tilde{M}$ for some point $p \in \tilde{M}$, then there exists a Riemannian splitting $\tilde{M}=\tilde{M}_{1} \times \tilde{M}_{2}$ such that the distribution $p \rightarrow \mathscr{N}(p)$ is the foliation of $\tilde{M}$ induced by $\tilde{M}_{1}$ and the sets $B_{p}$ are the maximal integral manifolds $\tilde{M}_{1} \times\left\{p_{2}\right\}$ of $\mathcal{N}$, where $p=\left(p_{1}, p_{2}\right)$. Moreover, if $\phi \in I(\tilde{M})$ leaves $X$ invariant, then $\phi$ leaves the splitting invariant.

The proof requires three preliminary results.

LEMMA 1. Let $X \subseteq \tilde{M}(\infty)$ be a closed involutive subset, and let $z \in \tilde{M}(\infty)$ and $p \in \tilde{M}$ be any points. Then the following statements are equivalent:

(1) $\measuredangle_{p}(z, x) \leq \pi / 2$ for all $x \in X$.

(2) $\measuredangle_{p}(z, x) \geq \pi / 2$ for all $x \in X$.

(3) $\measuredangle_{p}(z, x)=\pi / 2$ for all $x \in X$.

ProOF OF LEMMA 1. Let $p \in \tilde{M}$ and $z \in \tilde{M}(\infty)$ be as above. Given a point $x \in X$ we define $y=S_{p}(x)=\gamma_{p x}(-\infty)$. The point $y$ lies in $X$ since $X$ is involutive. The equivalence of the statements above is now immediate since $\pi=\measuredangle_{p}(z, x)+\measuredangle_{p}(z, y)$.

LEMMA 2. Let $\mathcal{N}(p)$ be a proper subspace of $T_{p} \tilde{M}$ for some point $p \in \tilde{M}$. Let $v \in \mathscr{N}(p)^{\perp}$ be any unit vector, and let $z=\gamma_{v}(\infty) \in \tilde{M}(\infty)$. Then $V(q, z)=$ $\gamma_{q z}^{\prime}(0) \in \mathscr{N}(q)^{\perp}$ for all $q \in \tilde{M}$.

ProOF OF LEMMA 2. We show first that $\measuredangle_{\gamma(t)}(z, x)=\pi / 2$ for all $t \in \mathbf{R}$ and all $x \in X$, where $\gamma(t)=\gamma_{v}(t)$. It suffices to consider the case $t \geq 0$ since $\gamma(-t)=\sigma(t)$ has initial velocity $-v \in \mathscr{N}(p)^{\perp}$. Fix $x \in X$. The assertion is true for $t=0$ since $\measuredangle_{p}(z, x)=\measuredangle\left(v, \gamma_{p x}^{\prime}(0)\right)=\pi / 2$ by the fact that $v \in \mathscr{N}(p)^{\perp}$. If $\theta(t)=\measuredangle_{\gamma(t)}(p, x)$ for $t>0$, then $\theta(t) \leq \pi-\measuredangle_{p}(z, x)=\pi / 2$ since the sum of the interior angles of a geodesic triangle of $\tilde{M}$ is at most $\pi$, even when one of the "vertices" lies in $\tilde{M}(\infty)$. Hence $\measuredangle_{\gamma(t)}(z, x)=\pi-\theta(t) \geq \pi / 2$ for all $t>0$. From Lemma 1 it now follows that $\measuredangle_{\gamma(t)}(z, x)=\pi / 2$ for all $t \in \mathbf{R}$ and all $x \in X$.

Now let $z^{*}=\gamma_{v}(-\infty)=\gamma_{-v}(\infty)$. We show that for every $x \in X$ the geodesic $\gamma_{p x}(\mathbf{R})$ is contained in $H(p, z) \cap H\left(p, z^{*}\right)$, the intersection of the horospheres through $p$ determined by $z$ and $z^{*}$ (see (1.2)). Fix $x \in X$. We showed above that $\measuredangle_{\gamma_{v}(t)}(z, x)=\measuredangle_{\gamma_{v}(t)}\left(z^{*}, x\right)=\pi / 2$ for all $t \in \mathbf{R}$ and all $x \in X$. It follows from Sublemma 5.4a of [E6, p. 78] that $\gamma_{v}(\mathbf{R})$ and $\gamma_{p x}[0, \infty]$ span a flat half plane in $\tilde{M}$ with boundary $\gamma_{v}(\mathbf{R})$. Hence $\gamma_{p x}[0, \infty] \subseteq H(p, z) \cap H\left(p, z^{*}\right)$ by the Euclidean geometry of this flat half plane. Since $y=\gamma_{p x}(-\infty) \in X$ it follows by the argument above that $\gamma_{p y}[0, \infty]=\gamma_{p x}[-\infty, 0] \subseteq H(p, z) \cap H\left(p, z^{*}\right)$. Hence $\gamma_{p x}(\mathbf{R}) \subseteq H(p, z) \cap H\left(p, z^{*}\right)$.

We now complete the proof of Lemma 2. Let $q \in \tilde{M}$ and $x \in X$ be given. Let $f_{z}$ be the Busemann function at $z$ such that $f_{z}(q)=0$. By the previous paragraph $f_{z}\left(\gamma_{p x} t\right) \equiv c$ in $\mathbf{R}$. Hence for $t \geq 0$ we have

$$
\begin{aligned}
f_{z}\left(\gamma_{q x} t\right) & =\left[f_{z}\left(\gamma_{q x} t\right)-f_{z}\left(\gamma_{p x} t\right)\right]+f_{z}\left(\gamma_{p x} t\right) \\
& \leq c+d\left(\gamma_{q x} t, \gamma_{p x} t\right) \leq c+d(p, q)
\end{aligned}
$$

by (1.2) and the fact that $\gamma_{p x}$ and $\gamma_{q x}$ are asymptotes. The convexity of $f_{z}$ implies that $t \rightarrow f_{z}\left(\gamma_{q x} t\right)$ is nonincreasing in $t$ and hence

$$
\cos \measuredangle_{q}(x, z)=-\left\langle\operatorname{grad} f_{z}(q), \gamma_{q x}^{\prime}(0)\right\rangle \geq 0
$$


by (1.2). This proves that $\measuredangle_{q}(z, x) \leq \pi / 2$ for all $q \in \tilde{M}$ and all $x \in X$ and equality holds by Lemma 1 . It now follows from the definition of $\mathscr{N}(q)$ that $V(q, z)=$ $\gamma_{q z}^{\prime}(0) \in \mathscr{N}(q)^{\perp}$ for all $q \in \tilde{M}$.

LEMMA 3. The following statements are equivalent:

(1) $B_{p}$ is a proper subset of all points $p$ of $\tilde{M}$.

(2) $B_{p}$ is a proper subset for some point $p$ of $\tilde{M}$.

(3) $\mathscr{N}(p)$ is a proper subspace of $T_{p} \tilde{M}$ for all points $p$ of $\tilde{M}$.

(4) $\mathscr{N}(p)$ is a proper subspace of $T_{p} \tilde{M}$ for some point $p$ of $\tilde{M}$.

Proof. The assertions (1) $\Rightarrow(2)$ and $(3) \Rightarrow(4)$ are obvious. We prove the assertions $(4) \Rightarrow(3),(3) \Rightarrow(1)$, and $(2) \Rightarrow(4)$.

PROOF OF $(4) \Rightarrow(3)$. Suppose that $\mathscr{N}(p)$ is a proper subspace of $T_{p} \tilde{M}$ for some point $p \in \tilde{M}$. Let $v \in \mathcal{N}(p)^{\perp}$ be any unit vector and let $z=\gamma_{v}(\infty)$. For any point $q \in \tilde{M}$ the unit vector $V(q, z)=\gamma_{q z}^{\prime}(0)$ lies in $\mathscr{N}(q)^{\perp}$ by Lemma 2 .

Proof OF $(3) \Rightarrow(1)$. Assume that $\mathscr{N}(p)$ is a proper subspace of $T_{p} \tilde{M}$ for all points $p \in \tilde{M}$, and let $p \in \tilde{M}$ be given. Let $v \in \mathscr{N}(p)^{\perp}$ be any unit vector and let $z=\gamma_{v}(\infty)$. In the proof of Lemma 2 we showed that for any $x \in X$ the geodesic $\gamma_{p x}(\mathbf{R})$ lies in $H(p, z) \subseteq B(p, z)=\left\{q \in \tilde{M}: f_{z}(q) \leq f_{z}(p)\right\}$, where $f_{z}$ is any Busemann function at $z$. Since $B(p, z)$ is a proper, closed, convex subset of $\tilde{M}$ it follows from the definition of $B_{p}$ that $B_{p} \subseteq B(p, z)$. Hence $B_{p}$ is a proper subset of $\tilde{M}$.

PROOF OF $(2) \Rightarrow(4)$. Suppose that $B_{p}$ is a proper subset for some point $p \in \tilde{M}$. Let $r$ be any point of $\tilde{M}-B_{p}$, and let $q \in B_{p}$ be the unique point in $B_{p}$ closest to $r$. Fix $x \in X$ and observe that $\gamma_{q x}[0, \infty] \subseteq B_{p}$; this follows from the fact that $\gamma_{p x}(\mathbf{R}) \subseteq B_{p}$ and $\gamma_{q x}(t)=\lim _{n \rightarrow \infty} \gamma_{n}(t)$ for all $t \in \mathbf{R}$, where $\gamma_{n}$ is the unique geodesic from $q$ to $\gamma_{p x}(n)$. If $z=\gamma_{q r}(\infty)$, then $\measuredangle_{q}(z, x)=\measuredangle_{q}(r, x) \geq \pi / 2$ for otherwise the function $t \rightarrow d\left(r, \gamma_{q x}(t)\right.$ would have a negative derivative at $t=0$, contradicting the definition of $q$. This shows that $\measuredangle_{q}(z, x) \geq \pi / 2$ for all $x \in X$ and equality follows by Lemma 1 . Hence if $v=\gamma_{q z}^{\prime}(0)$, then $v \in \mathscr{N}(q)^{\perp}$ and $\mathscr{N}(q)$ is a proper subspace of $T_{q} \tilde{M}$.

PROOF OF THEOREM A. The first assertion is contained in Lemma 3 . We now prove assertion (2), assuming that $\mathscr{N}(p)$ is a proper subspace of $T_{p} \tilde{M}$ for one and hence all points $p$ of $\tilde{M}$ by Lemma 3 .

We show first that the dimension of $\mathcal{N}(p)$ is constant in $\tilde{M}$. By the connectedness of $\tilde{M}$ it suffices to show that the dimension of $\mathscr{N}(p)$ is a locally constant function of $p$. We do this in two steps.

(a) $\operatorname{dim} \mathscr{N}(q) \geq \operatorname{dim} \mathscr{N}(p)$ for all $q$ near $p$.

(b) $\operatorname{dim} \mathscr{N}(q) \leq \operatorname{dim} \mathscr{N}(p)$ for all $q$ near $p$.

To prove (a) we choose points $x_{1}, x_{2}, \ldots, x_{N} \in X$ so that the unit vectors $\left\{V\left(p, x_{i}\right)\right.$ : $1 \leq i \leq N\}$ are a basis of $\mathcal{N}(p)$. If $q \in \tilde{M}$ is sufficiently close to $p$, then the vectors $\left\{V\left(q, x_{i}\right): 1 \leq i \leq N\right\}$ remain linearly independent elements of $\mathscr{N}(q)$, which proves (a).

Now choose points $y_{1}, \ldots, y_{r}$ in $\tilde{M}(\infty)$ so that $\left\{V\left(p, y_{j}\right): 1 \leq j \leq r\right\}$ is a basis for $\mathscr{N}(p)^{\perp}$. If $q \in \tilde{M}$ is arbitrary, then $\left\{V\left(q, y_{j}\right) \in \mathscr{N}(q)^{\perp}\right.$ for each $1 \leq j \leq r$ 
by Lemma 2, and if $q$ is sufficiently close to $p$, then these vectors remain linearly independent, which proves (b).

A $C^{1}$ distribution $\mathscr{F}$ in $\tilde{M}$ is said to be parallel if for any $C^{1}$ curve $\gamma:[a, b] \rightarrow \tilde{M}$ we have

$$
\mathscr{F}(\gamma(b)) \text { is the parallel translate along } \gamma \text { of } \mathscr{F}(\gamma(a)) \text {. }
$$

It is easy to see that a $C^{1}$ distribution $\mathscr{F}$ is parallel if and only if for any vector $v \in T_{p} \tilde{M}$ and any $C^{1}$ vector field $Z$ tangent to $\mathscr{F}$ near $p$ we have $\nabla_{v} Z \in \mathscr{F}(p)$.

Let $p \in \tilde{M}$ be given. By the discussion above we may choose a neighborhood 0 of $p$ in $\tilde{M}$, points $x_{1}, \ldots, x_{N}$ in $X$ and $y_{1}, \ldots, y_{r}$ in $\tilde{M}(\infty)$ such that $\left\{V\left(q, x_{i}\right): 1 \leq\right.$ $i \leq N\}$ and $\left\{V\left(q, y_{j}\right): 1 \leq j \leq r\right\}$ are bases for $\mathscr{N}(q)$ and $\mathscr{N}(q)^{\perp}$ for all $q \in 0$. Let $V_{i}, W_{j}$ be those unit vector fields in $\tilde{M}$ defined by $V_{i}(q)=V\left(q, x_{i}\right)$ and $W_{j}(q)=$ $V\left(q, y_{j}\right)$. The distribution $\mathcal{N}$ is $C^{1}$ since the vector fields $\left\{-V_{i}\right\}$ are the gradients of $C^{2}$ Busemann functions at $x_{i}$ by the discussion in (1.2).

We show

$$
\nabla_{V_{i}} W_{j} \equiv 0 \quad \text { and } \quad \nabla_{W_{j}} V_{i} \equiv 0 \text { in } \tilde{M} .
$$

By definition the vector field $V_{i}$ is tangent to $\mathscr{N}$ at all points of $\tilde{M}$ for every $1 \leq i \leq N$, and by Lemma 2 the vector field $W_{j}$ is tangent to $\mathscr{N}^{\perp}$ at all points of $\tilde{M}$ for every $1 \leq j \leq r$. Fixing $1 \leq i \leq N$ and $1 \leq j \leq r$ we conclude by Sublemma 5.4a of [E6, p. 78] that for every point $q \in \tilde{M}$ the orthogonal geodesics $\gamma_{q x_{i}}(\mathbf{R})$ and $\gamma_{q y_{j}}[0, \infty]$ determine a flat half plane in $\tilde{M}$ whose boundary is $\gamma_{q x_{i}}(\mathbf{R})$. Hence the vector field $t \rightarrow W_{j}(\gamma t)$ is parallel on $\gamma(\mathbf{R})=\gamma_{q x_{i}}(\mathbf{R})$, and it follows that $\left(\nabla_{V_{i}} W_{j}\right)(q)=0$. Similarly $\nabla_{W_{i}} V_{j} \equiv 0$ in $\tilde{M}$.

To prove that $\mathscr{N}$ is a parallel distribution in $\tilde{M}$ it suffices by $(*)$ and the discussion above to show that $\left(\nabla_{V_{i}} V_{j}\right)(q) \in \mathcal{N}(q)$ for all $1 \leq i, j \leq N$ and all $q$ in the given neighborhood 0 of $p$. For $1 \leq k \leq r$ and $1 \leq i, j \leq N$ we have

$$
\left\langle\nabla_{V_{i}} V_{j}, W_{k}\right\rangle=V_{i}\left\langle V_{j}, W_{k}\right\rangle-\left\langle V_{j}, \nabla_{V_{i}} W_{k}\right\rangle=0
$$

by (*) and the fact that $V_{i}, V_{j}$ are orthogonal to $W_{k}$ in $\tilde{M}$. Since $\left\{W_{1}(q), \ldots, W_{r}(q)\right\}$ is a basis for $\mathscr{N}(q)^{\perp}$ for all $q \in 0$ it follows that $\mathscr{N}$ is a parallel distribution in $\tilde{M}$.

Since $\mathscr{N}$ is parallel the de Rham theorem implies that $\tilde{M}$ splits as a Riemannian product $\tilde{M}_{1} \times \tilde{M}_{2}$ such that the foliations of $\tilde{M}$ induced by $\tilde{M}_{1}, \tilde{M}_{2}$ are $\mathscr{N}, \mathscr{N}^{\perp}$ respectively [KN]. If $F_{p}$ denotes the maximal integral manifold of $\mathscr{N}$ passing through $p$, then $F_{p}$ is a complete totally geodesic submanifold of $\tilde{M}$. We show that $F_{p}=B_{p}$. By definition $\mathscr{N}(p)=T_{p} F_{p}$ and hence $\gamma_{p x}(\mathbf{R}) \subseteq F_{p}$ for all $x \in X$ since $\gamma_{p x}^{\prime}(0) \in \mathscr{N}(p)$ for all $x \in X$. It follows that $B_{p} \subseteq F_{p}$ since $F_{p}$ is a closed convex subset of $\tilde{M}$.

Suppose that $B_{p}$ is a proper subset of $F_{p}$, and let $r$ be any point in $F_{p}-B_{p}$. Let $q \in B_{p}$ be the unique point in $B_{p}$ closest to $r$, and let $v=\gamma_{q r}^{\prime}(0)$. The proof of assertion (2) $\Rightarrow(4)$ in Lemma 3 implies that $v \in \mathscr{N}(q)^{\perp}$, and $v \in T_{q} F_{p}$ since $r$ lies in $F_{p}$. On the other hand $\mathscr{N}(q)=T_{q} F_{p}$ since $F_{p}$ is complete and totally geodesic and $\mathscr{N}$ is parallel in $\tilde{M}$. This implies that $v$ is a unit vector in $\mathscr{N}(q) \cap \mathscr{N}(q)^{\perp}$, a contradiction that implies $B_{p}=F_{p}$. 
Finally, if $\varphi \in I(\tilde{M})$ leaves invariant the involutive subset $X \subseteq \tilde{M}(\infty)$, then $d \varphi \mathscr{N}(p)=\mathscr{N}(\varphi p)$ for every $p \in \tilde{M}$ by the definition of $\mathscr{N}$. Hence $d \varphi \mathscr{N}(p)^{\perp}=$ $\mathscr{N}(\varphi p)^{\perp}$ for every $p \in \tilde{M}$. By definition (see (1.3)) it follows that $\varphi$ leaves invariant the splitting $\tilde{M}=\tilde{M}_{1} \times \tilde{M}_{2}$, which completes the proof of Theorem A.

4. Applications. In this section and the next we shall use Theorem A to derive some new results and to simplify the proofs of some known results. Our general theme is the study of groups $N \subseteq I(\tilde{M})$ whose normalizer in $I(\tilde{M})$ satisfies the duality condition. We begin with the following useful

LEMMA. Let $N \subseteq I(\tilde{M})$ be a nonidentity subgroup whose normalizer $\Gamma$ in $I(\tilde{M})$ satisfies the duality condition and whose centralizer $Z$ in $I(\tilde{M})$ is not the identity. Then either

(1) $Z \subseteq C(\tilde{M})$, the subgroup of Clifford translations of $\tilde{M}$ or

(2) There exists a nontrivial Riemannian splitting $\tilde{M}=\tilde{M}_{1} \times \tilde{M}_{2}$ that is invariant under $\Gamma$ and $Z$ and such that

(a) $N \subseteq I\left(\tilde{M}_{1}\right) \times\{1\}$,

(b) $p_{1}(Z) \subseteq C\left(\tilde{M}_{1}\right)$, where $p_{1}$ denotes the projection homeomorphism into $I\left(\tilde{M}_{1}\right)$.

From the discussion in (1.4) we then obtain the following

COROLLARY. Let $N \subseteq I(\tilde{M})$ be a nonidentity subgroup whose normalizer $\Gamma$ in $I(\tilde{M})$ satisfies the duality condition. If $\tilde{M}$ is irreducible, then the centralizer $Z$ of $N$ in $I(\tilde{M})$ is the identity.

ProOF OF THE LEMMA. By Corollary $2.5 \mathrm{~b}$ the limit set $L(N)$ is a nonempty, closed, involutive subset of $\tilde{M}(\infty)$. Let $B_{p}$ denote the closed convex hull of $\left\{\gamma_{p x}(\mathbf{R})\right.$ : $x \in L(N)\}$ for each point $p$ of $\tilde{M}$. If $\varphi \in Z$, then $d_{\varphi}(\psi p)=d_{\varphi}(p)$ for all $\psi \in N$ since $\varphi \psi=\psi \varphi$, and by the convexity of $d_{\varphi}$ it follows that $d_{\varphi} \leq d_{\varphi}(p)$ on $\gamma_{p x}[0, \infty)$ for every $x \in L(N)$. Since $L(N)$ is involutive it follows that $d_{\varphi} \leq d_{\varphi}(p)$ on $\gamma_{p x}(\mathbf{R})$ for every $x \in L(N)$ and hence $d_{\varphi} \leq d_{\varphi}(p)$ on $B_{p}$. If $B_{p}=\tilde{M}$ for some point $p$ of $\tilde{M}$, then $Z \subseteq C(\tilde{M})$ by the discussion above in (1.4).

Suppose now that $Z \nsubseteq C(\tilde{M})$ and $B_{p}$ is a proper subset of $\tilde{M}$ for every point $p$ of $\tilde{M}$. By Theorem A in the previous section there exists a Riemannian splitting $\tilde{M}=M_{1}^{*} \times M_{2}^{*}$ such that $B_{p}$ is the maximal integral manifold through $p$ of the foliation of $\tilde{M}$ induced by $M_{1}^{*}$.

We shall show that the splitting $\tilde{M}=M_{1}^{*} \times M_{2}^{*}$ is invariant under both $\Gamma$ and $Z$ and moreover

(i) $p_{1}^{*}(Z) \subseteq C\left(M_{1}^{*}\right)$, where $p_{1}^{*}: Z \rightarrow I\left(M_{1}^{*}\right)$ is the projection homomorphism,

(ii) $L(N) \subseteq M_{1}^{*}(\infty)$.

We shall then show that a $\Gamma$ and $Z$ invariant splitting $\tilde{M}=M_{1}^{*} \times M_{2}^{*}$ satisfies case (2) of the lemma if it satisfies properties (i) and (ii) above and maximizes the dimension of $M_{1}^{*}$ with respect to these properties.

Assertion (ii) follows from the definitions of $B_{p}$ and $M_{1}^{*}$. The splitting $\tilde{M}=M_{1}^{*} \times$ $M_{2}^{*}$ is invariant under both $\Gamma$ and $Z$ by Theorem A since both groups normalize $N$ and hence leave $L(N)$ invariant. Therefore for every element $\varphi$ in $\Gamma$ or $Z$ we may write $\varphi=\left(\varphi_{1}, \varphi_{2}\right)$, where $\varphi_{i}=p_{i}^{*}(\varphi) \in I\left(M_{i}^{*}\right)$ and $p_{i}^{*}$ is the projection homomorphism into $I\left(M_{i}^{*}\right)$ for $i=1,2$. If $\varphi=\left(\varphi_{1}, \varphi_{2}\right) \in Z$, then $d_{\varphi_{1}}$ is bounded 
above on $M_{1}^{*}$ since $d_{\varphi} \leq d_{\varphi}(p)$ on $B_{p}$ for every point $p \in \tilde{M}$ by the discussion above. Hence $\varphi_{1} \in C\left(M_{1}^{*}\right)$ by the discussion in (1.4), which proves assertion (i) above.

Now let $\tilde{M}=M_{1}^{*} \times M_{2}^{*}$ be a Riemannian splitting that is invariant under $\Gamma$ and $Z$, satisfies (i) and (ii) above and maximizes the dimension of $M_{1}^{*}$ with respect to these properties. We show that $p_{2}^{*}(N)=\{1\}$, which will complete the proof of the lemma. Let $\Gamma_{2}, N_{2}$ and $Z_{2}$ denote $p_{2}^{*}(\Gamma), p_{2}^{*}(N)$ and $p_{2}^{*}(Z)$ respectively. By Proposition 1.6b $\Gamma_{2}$ satisfies the duality condition in $M_{2}^{*}$. Our assumption from above is that $Z \nsubseteq C(\tilde{M})$ and in view of (i) this implies that $Z_{2} \neq\{1\}$ and $Z_{2} \nsubseteq C\left(M_{2}^{*}\right)$.

We assume that $N_{2} \neq\{1\}$ and obtain a contradiction. Since $N_{2}$ is normalized by $\Gamma_{2}$ and centralized by $Z_{2}$ we may apply the arguments above to $\Gamma_{2}, N_{2}$ and $Z_{2}$ and obtain a nontrivial Riemannian splitting $M_{2}^{*}=\tilde{M}_{\alpha} \times \tilde{M}_{\beta}$ that is invariant under $\Gamma_{2}$ and $Z_{2}$ and such that

(i)' $p_{\alpha}\left(Z_{2}\right) \subseteq C\left(\tilde{M}_{\alpha}\right)$, where $p_{\alpha}: \Gamma_{2} \rightarrow I\left(\tilde{M}_{\alpha}\right)$ denotes the projection homomorphism,

(ii) ${ }^{\prime} L\left(N_{2}\right) \subseteq \tilde{M}_{\alpha}(\infty)$.

Now let $\tilde{M}_{1}=M_{1}^{*} \times \tilde{M}_{\alpha}$ and $\tilde{M}_{2}=\tilde{M}_{\beta}$. Then the splitting $\tilde{M}=\tilde{M}_{1} \times \tilde{M}_{2}$ is invariant under $\Gamma$ and $Z$ and satisfies

(i) $p_{1}(Z) \subseteq C\left(\tilde{M}_{1}\right)$, where $p_{1}: \Gamma \rightarrow I\left(\tilde{M}_{1}\right)$ denotes the projection homomorphism,

(ii) $L(N) \subseteq \tilde{M}_{1}(\infty)$.

Since $\operatorname{dim} \tilde{M}_{1}>\operatorname{dim} M_{1}^{*}$ we obtain a contradiction to the hypothesis that $M_{1}^{*}$ has maximal dimension with respect to $\Gamma$ and $Z$ invariant splittings of $\tilde{M}$ that satisfy (i) and (ii). This proves that $N_{2}=p_{2}^{*}(N)=\{1\}$, which completes the proof of the lemma.

Our next result is Theorem 2.4 of $[\mathbf{C E}]$.

THEOREM B. Let $N \subseteq I(\tilde{M})$ be an abelian group whose normalizer $\Gamma$ in $I(\tilde{M})$ satisfies the duality condition. Then $N \subseteq C(\tilde{M})$.

Proof. In the notation of the lemma note that $N \subseteq Z$ since $N$ is abelian. In either of the two cases of the lemma it is now clear that $N \subseteq C(\tilde{M})$.

The next result is a part of Theorem 4.2 of [CE].

THEOREM C. Let $\Gamma \subset I(\tilde{M})$ satisfy the duality condition and suppose that the centralizer $Z$ of $\Gamma$ in $I(\tilde{M})$ is not the identity. Then there exists a Riemannian splitting $\tilde{M}=M_{0}^{*} \times M_{1}^{*}$ that is invariant under $\Gamma$ and $Z$ and such that

(1) $M_{0}^{*}$ is a Euclidean space of positive dimension,

(2) $Z=C\left(M_{0}^{*}\right) \times\{1\}$,

(3) $p_{0}^{*}(\Gamma) \subseteq C\left(M_{0}^{*}\right)$,

(4) $p_{1}^{*}(\Gamma)$ has trivial centralizer in $I\left(M_{1}^{*}\right)$.

Here $p_{0}^{*}, p_{1}^{*}$ denote the projection homomorphisms into $I\left(M_{0}^{*}\right), I\left(M_{1}^{*}\right)$ respectively.

ProOF. We let $N=\Gamma$ in the notation of the lemma above. Possibility 2(a) of the Lemma does not arise since $\Gamma$ satisfies the duality condition and hence $Z \subseteq C(\tilde{M})$ by the Lemma. By the discussion in (1.4) we can write $\tilde{M}$ as a Riemannian product $M_{0}^{*} \times M_{1}^{*}$, where $M_{0}^{*}$ is a Euclidean space of positive dimension, $L(Z)=M_{0}^{*}(\infty)$ 
and $\varphi=\left(\varphi_{0}\right.$, id) for every $\varphi \in Z$ with $\varphi_{0}$ a translation of $M_{0}^{*}$. It follows that $p_{0}^{*}(\Gamma) \subseteq C\left(M_{0}^{*}\right)$ since $\Gamma$ commutes with $Z$, and hence $Z=C\left(M_{0}^{*}\right) \times\{1\}$, which proves (2) and (3). Finally (4) follows from (2).

The next result except for assertion (4) is Theorem 1 of $[\mathbf{S}]$.

THEOREM D. Let $\Gamma \subseteq I(\tilde{M})$ satisfy the duality condition, and suppose that $\Gamma=A \cdot B$, where $A$ and $B$ are commuting subgroups of $\Gamma$. Then there exists a $\Gamma$-invariant Riemannian splitting $\tilde{M}=\tilde{M}_{0} \times \tilde{M}_{1} \times \tilde{M}_{2}$ such that

(1) $\tilde{M}_{0}$ is a Euclidean space of dimension $r \geq 0$ and $p_{0}(\Gamma) \subseteq C\left(\tilde{M}_{0}\right)$.

(2) $C\left(\tilde{M}_{0}\right) \times$ id $\times$ id is the centralizer $Z$ of $\Gamma$ in $I(\tilde{M})$.

(3) (a) If $\varphi \in A$, then $\varphi=\left(\varphi_{0}, \varphi_{1}\right.$, id), where $\varphi_{0} \in C\left(\tilde{M}_{0}\right)$ and $\varphi_{1} \in I\left(\tilde{M}_{1}\right)$.

(b) If $\varphi \in B$, then $\varphi=\left(\varphi_{0}\right.$, id, $\left.\varphi_{2}\right)$, where $\varphi_{0} \in C\left(\tilde{M}_{0}\right)$ and $\varphi_{2} \in I\left(\tilde{M}_{2}\right)$.

(4) If $\Gamma$ is discrete, then $p_{1}(\Gamma)=p_{1}(A)$ and $p_{2}(\Gamma)=p_{2}(B)$ are discrete and have trivial centralizer in $I\left(\tilde{M}_{1}\right), I\left(\tilde{M}_{2}\right)$ respectively.

Again, $p_{i}: \Gamma \rightarrow I\left(\tilde{M}_{i}\right)$ denotes the projection homomorphism for $i=0,1,2$. Any one of the factors in the results above may be absent and $\tilde{M}=\tilde{M}_{0}$ is also possible.

Proof. We shall consider only the case that $Z$, the centralizer of $\Gamma$ in $I(\tilde{M})$, is nontrivial. If $Z=\{1\}$, then the factor $\tilde{M}_{0}$ is absent in the final splitting of $\tilde{M}$ and we proceed as below. Assuming that $Z \neq\{1\}$ we use Theorem $\mathrm{C}$ to obtain a $\Gamma$-invariant splitting $\tilde{M}=M_{0}^{*} \times M_{1}^{*}$, where $M_{0}^{*}$ is a Euclidean space of positive dimension and $Z=C\left(M_{0}^{*}\right) \times\{1\}$. If $p_{i}^{*}: \Gamma \rightarrow I\left(M_{i}^{*}\right)$ denotes the projection homomorphism for $i=0,1$, then clearly $p_{0}^{*}(\Gamma) \subseteq C\left(M_{0}^{*}\right)$. Let $\Gamma_{1}, A_{1}, B_{1}$ denote $p_{1}^{*}(\Gamma), p_{1}^{*}(A), p_{1}^{*}(B)$ respectively. Then $\Gamma_{1}=A_{1} \cdot B_{1}$, where $A_{1}$ and $B_{1}$ commute. By the characterization of $Z$ the group $\Gamma_{1}$ has a trivial centralizer in $M_{1}^{*}$. We consider only the case that $A_{1}$ and $B_{1}$ are both nonidentity subgroups of $I\left(M_{1}^{*}\right)$. The remaining cases are handled similarly and correspond to the absence of one or both of the factors $\tilde{M}_{1}, \tilde{M}_{2}$ in the statement of the theorem. We now apply the lemma at the beginning of this section to the group $N=A_{1}$ and note that $1 \neq B_{1} \subseteq Z_{1}$, where $Z_{1}$ denotes the centralizer of $A_{1}$ in $I\left(M_{1}^{*}\right)$. If $Z_{1} \subseteq C\left(M_{1}^{*}\right)$, then $B_{1}$ is abelian and hence lies in the center of $\Gamma_{1}$, contradicting the fact that $\Gamma_{1}$ has trivial centralizer in $I\left(M_{1}^{*}\right)$. Hence from the lemma of this section we obtain a nontrivial Riemannian splitting $M_{1}^{*}=\tilde{M}_{\alpha} \times \tilde{M}_{\beta}$ that is invariant under $\Gamma_{1}$ and $Z_{1}$ and such that $A_{1} \subseteq I\left(\tilde{M}_{\alpha}\right) \times\{1\}$ and $p_{\alpha}\left(Z_{1}\right) \subseteq C\left(\tilde{M}_{\alpha}\right)$, where $p_{\alpha}$ is the projection homomorphism into $I\left(\tilde{M}_{\alpha}\right)$. Since $p_{\alpha}\left(Z_{1}\right)$ is abelian and contains $p_{\alpha}\left(B_{1}\right)$ it follows that $p_{\alpha}\left(B_{1}\right)$ commutes with both $A_{1}$ and $B_{1}$ and hence with $\Gamma_{1}=A_{1} \cdot B_{1}$. Therefore $p_{\alpha}\left(B_{1}\right)=\{1\}$ since $\Gamma_{1}$ has trivial centralizer in $I\left(\tilde{M}_{1}\right)$. If we define $\tilde{M}_{0}=M_{0}^{*}$, $\tilde{M}_{1}=\tilde{M}_{\alpha}$ and $\tilde{M}_{2}=\tilde{M}_{\beta}$, then the splitting $\tilde{M}=\tilde{M}_{0} \times \tilde{M}_{1} \times \tilde{M}_{2}$ is invariant under $\Gamma$ and satisfies assertions (1), (2) and (3) of Theorem D.

We prove assertion (4) of the theorem. We shall need the following.

LEMMA. Let $N \subseteq I(\tilde{M})$ be a discrete group and let $G$ denote the normalizer of $N$ in $I(\tilde{M})$. Then $G_{0}$, the connected component of $G$ that contains the identity, lies in the centralizer of $N$ in $I(\tilde{M})$.

PROOF OF THE LEMMA. Let $\varphi \in N$ be given. If $g(t)$ is a curve in $G$ that starts at the identity, then $\varphi(t)=g(t) \varphi g(t)^{-1}$ is a curve in $N$ that starts at $\varphi$. The discreteness of $N$ implies that $\varphi(t)=\varphi$ for all $t$ and hence $\exp (\mathfrak{G})$ centralizes 
$N$, where $\mathfrak{G}$ is the Lie algebra of $G$ and exp: $\mathfrak{G} \rightarrow G$ is the exponential map. The result follows since $\exp (\mathfrak{G})$ contains a neighborhood of the identity in $G$ and any neighborhood of the identity in $G$ generates $G_{0}$.

Assume that the group $\Gamma$ in the statement of the theorem is discrete. If $p_{1}(\Gamma) \subseteq$ $I\left(\tilde{M}_{1}\right)$ is not discrete, then $\overline{\left(p_{1}(\Gamma)\right)_{0}}$ centralizes $\Gamma$ by the lemma just proved, which contradicts assertion (2) of the theorem. Therefore $p_{1}(\Gamma)$ is discrete in $I\left(\tilde{M}_{1}\right)$ and similarly $p_{2}(\Gamma)$ is discrete in $I\left(\tilde{M}_{2}\right)$. Finally it follows from assertion $(3)$ of the theorem that $p_{1}(\Gamma)=p_{1}(A)$ and $p_{2}(\Gamma)=p_{2}(B)$. The fact that $p_{1}(\Gamma)$ has trivial centralizer in $I\left(\tilde{M}_{i}\right)$ for $i=1,2$ is a consequence of assertion (2).

Our next result is essentially the main theorem in $\S 3$ of [E3] but the proof here is shorter and conceptually simpler.

THEOREM E. Let $\tilde{M}$ have a trivial Euclidean de Rham factor. Let $G \subseteq I(\tilde{M})$ be a closed, connected Lie subgroup of positive dimension whose normalizer $\Gamma$ in $I(\tilde{M})$ satisfies the duality condition. Then there exists a $\Gamma$-invariant Riemannian splitting $\tilde{M}=\tilde{M}_{1} \times \tilde{M}_{2}$ such that

(1) $\tilde{M}_{1}$ is a symmetric space of noncompact type.

(2) $G=I_{0}\left(\tilde{M}_{1}\right) \times\{1\}$.

PROOF. We shall need three preliminary results.

LEMMA E1. $G$ is a semisimple Lie group with trivial center and no compact normal subgroups except the identity.

LEMMA E2. If $L(G)=\tilde{M}(\infty)$, then $\tilde{M}$ is a symmetric space of noncompact type and $G=I_{0}(\tilde{M})$.

LEMMA E3. Let $\tilde{M}$ be an arbitrary, complete, simply connected manifold of nonpositive sectional curvature. Let $G \subseteq I(\tilde{M})$ be a closed, connected Lie subgroup of positive dimension that is semisimple with finite center and has no compact normal subgroups except the identity. Let $\Gamma$ denote the normalizer of $G$ in $I(\tilde{M})$ and let $Z_{\Gamma}(G)=\{\varphi \in \Gamma: \varphi g=g \varphi$ for all $g \in G\}$. Then $\Gamma_{0}=G \cdot Z_{\Gamma}(G)$ has finite index in $\Gamma$.

ProOF OF THE LEMMAS. Lemma E1 is Lemma 3.1 of [E3] and the proof of Lemma E2 is contained in the proof of Lemma 3.4 of [E3]. We prove Lemma E3 in two steps.

Step 1. Let $\Gamma, G$ be as in Lemma E3 and let $K$ be a maximal compact subgroup of $G$. Let $N_{\Gamma}(K)$ denote the normalizer of $K$ in $\Gamma$. Then

(1) $\Gamma=G \cdot N_{\Gamma}(K)$.

(2) If $\mathfrak{G}, \mathfrak{K}$ denote the Lie algebras of $G, K$ then $\operatorname{Aut}_{\mathfrak{K}}(\mathfrak{G})=\{\varphi \in \operatorname{Aut}(\mathfrak{G})$ : $\varphi(\mathfrak{K}) \subseteq \mathfrak{K}\}$ is a compact subgroup of $\operatorname{Aut}(\mathfrak{G})$.

REMARK. With regard to (2) we recall that Aut($(\mathfrak{G )}$ denotes the group of Lie algebra automorphisms of $\mathfrak{G}$. Since $\operatorname{Ad}(G)$ is a connected Lie subgroup of $\operatorname{Aut}(\mathfrak{G})$ whose Lie algebra is $\operatorname{ad}(\mathfrak{G})=\{\operatorname{ad} X: X \in \mathfrak{G}\}$ it follows from the conditions on $G$ that $\operatorname{Ad}(G)$ is the connected component of $\operatorname{Aut}(\mathfrak{G})$ that contains the identity; see for example Corollary 6.5 of [H, p. 122].

PROOF OF STEP 1. If $X$ denotes the coset space $G / K$, then $X$ equipped with a suitable left invariant metric becomes a Riemannian symmetric space of noncompact type; see for example Proposition 3.6 of [H, p. 178] and Theorem 3.1 
of [H, p. 205]. The group $K$ has a fixed point $p$ in $X$ by the Cartan fixed point theorem, and in fact $K=\{\varphi \in G: \varphi(p)=p\}$ since the latter is a compact subgroup of $I(\tilde{M})$. Since $G$ acts transitively on $X$ all maximal compact subgroups of $G$ are conjugate by elements of $G$.

We prove (1). Given an element $\varphi \in \Gamma$ the subgroup $\varphi K \varphi^{-1}$ is a maximal compact subgroup of $\varphi G \varphi^{-1}=G$ and hence $\varphi K \varphi^{-1}=g K g^{-1}$ for some $g \in G$ by the remarks above. If $\psi=g^{-1} \varphi$, then $\psi \in N_{\Gamma}(K)$ and hence $\varphi=g \psi \in$ $G \cdot N_{\Gamma}(K)$.

We prove (2). For a discussion of definitions and facts relevant to this proof see, for example, [E7, pp. 121-133]. Let $\mathfrak{G}=\mathfrak{K}+\mathfrak{P}$ be the Cartan decomposition of $G$ induced by $\mathfrak{K}$, where $\mathfrak{K}$ is the Lie algebra of $K$ and $\mathfrak{P}$ is the orthogonal complement of $\mathfrak{K}$ in $\mathfrak{G}$ with respect to the Killing form $B$ of $\mathfrak{G}$. It is known that $B$ is positive definite on $\mathfrak{P}$ and negative definite on $\mathfrak{K}$. If $\varphi \in \operatorname{Aut}_{\mathfrak{K}}(\mathfrak{G})$, then $\varphi$ preserves $B[\mathbf{H}$, p. 121] and hence $\varphi(\mathfrak{P}) \subseteq \mathfrak{P}$ since $\varphi(k) \subseteq \mathfrak{K}$. It follows that $\varphi$ preserves the positive definite inner product $\Phi$ on $\mathfrak{G}$ given by $\Phi(k, \mathfrak{P})=0, \Phi=B$ on $\mathfrak{P}$ and $\Phi=-B$ on $\mathfrak{K}$. This proves (2) since $\operatorname{Aut}_{\mathfrak{K}}(\mathfrak{G})$ is a closed subgroup of the orthogonal group $O(\mathfrak{G}, \Phi)$, which is compact.

Step 2. We prove that $N_{\Gamma}(K) \cap\left[G \cdot Z_{\Gamma}(G)\right]$ has finite index in $N_{\Gamma}(K)$. In combination with assertion (1) of Step 1 this will complete the proof of Lemma E3.

We begin by defining a homomorphism $\rho: \Gamma \rightarrow \operatorname{Aut}(\mathfrak{G})$ by setting

$$
\varphi e^{t X} \varphi^{-1}=e^{t \rho(\varphi) X}
$$

for all $\varphi \in \Gamma, X \in \mathfrak{G}$ and $t \in \mathbf{R}$. The left-hand side of the expression above is a 1-parameter subgroup of $G$ for every $\varphi \in \Gamma$ and $X \in \mathfrak{G}$, and the subgroup lies in $\mathfrak{K}$ if $\varphi \in N_{\Gamma}(K)$ and $X \in \mathfrak{K}$. Hence we obtain by restriction a homomorphism

$$
\rho: N_{\Gamma}(K) \rightarrow \operatorname{Aut}_{\mathfrak{K}}(\mathfrak{G}) .
$$

Now let $N_{0}$ denote $N_{\Gamma}(K) \cap\left[G \cdot Z_{\Gamma}(G)\right]$ and suppose that the assertion of Step 2 is false. Then $N_{0}$ has infinite index in $N_{\Gamma}(K)$ and we may choose a sequence $\left\{\varphi_{k}\right\} \subseteq N_{\Gamma}(K)$ so that the cosets $\left\{\varphi_{k} N_{0}\right\}$ are all distinct. By the remark preceding the proof of Step 1 we can find a neighborhood $0 \subseteq \operatorname{Aut}(\mathfrak{G})$ of the identity such that $0 \subseteq \operatorname{Ad}(G)$. Since $\left\{\rho\left(\varphi_{k}\right)\right\}$ is a bounded sequence in $\operatorname{Aut}_{\mathfrak{K}}(\mathfrak{G})$ by assertion (2) of Step 1, we can find distinct integers $n$ and $m$ such that $\rho\left(\varphi_{n}^{-1} \varphi_{m}\right) \in 0$. Choose $g \in G$ so that $\rho\left(\varphi_{n}^{-1} \varphi_{m}\right)=\operatorname{Ad}(g)$, and let $\xi=g^{-1} \varphi_{n}^{-1} \varphi_{m}$. From the definition of $\rho$ and Ad it follows that $\xi e^{t X} \xi^{-1}=e^{t X}$ for all $X \in \mathfrak{G}$ and all $t \in \mathbf{R}$. Hence $\xi \in Z_{\Gamma} G$ since $\exp (\mathfrak{G})$ contains a neighborhood $0^{*} \subseteq G$ of the identity and $0^{*}$ generates $G$. Therefore $\varphi_{n}^{-1} \varphi_{m}=g \xi \in N_{0}$ and it follows that $\varphi_{n} N_{0}=\varphi_{m} N_{0}$, contradicting the way in which the sequence $\left\{\varphi_{k}\right\}$ was chosen. Therefore $N_{0}$ has finite index in $N_{\Gamma}(K)$, which completes the proof of Lemma E3 as indicated above.

ProOF OF THEOREM E. Let $\Gamma, G$ be as in the statement of the theorem, and let $\Gamma_{0}=G \cdot Z_{\Gamma}(G)$. By Lemma $\mathrm{E} 3 \Gamma_{0}$ has finite index in $\Gamma$ and hence $\Gamma_{0}$ satisfies the duality condition by Proposition 1.6b. By hypothesis $\tilde{M}$ has no Euclidean de Rham factor and hence it follows from Theorem D that there exists a $\Gamma_{0}$-invariant Riemannian splitting $\tilde{M}=\tilde{M}_{1} \times \tilde{M}_{2}$ such that $G \subseteq I\left(\tilde{M}_{1}\right) \times\{1\}$ and $Z_{\Gamma}(G) \subseteq$ $\{1\} \times I\left(\tilde{M}_{2}\right)$. If $p_{1}: \Gamma_{0} \rightarrow I\left(\tilde{M}_{1}\right)$ denotes the projection homomorphism, then $G=p_{1}\left(\Gamma_{0}\right)$ and hence $G$ satisfies the duality condition in $\tilde{M}_{1}$ by Proposition $1.6 \mathrm{~b}$. 
Regarding $G$ as a subgroup of $I\left(\tilde{M}_{1}\right)$ and applying Lemma E2 we conclude that $\tilde{M}_{1}$ is a symmetric space of noncompact type and $G=I_{0}\left(\tilde{M}_{1}\right)$.

As a corollary of the result just proved we obtain

THEOREM F. Let $\tilde{M}=\tilde{M}_{0} \times \tilde{M}_{1} \times \cdots \times \tilde{M}_{k}$ be the de Rham decomposition of $\tilde{M}$, where $\tilde{M}_{0}$ is the Euclidean factor of $\tilde{M}$ and $\left\{\tilde{M}_{i}: 1 \leq i \leq k\right\}$ are the irreducible non-Euclidean factors of $\tilde{M}$. Let $\Gamma \subseteq I(\tilde{M})$ be a subgroup that leaves the splitting invariant and satisfies the duality condition. Let $p_{i}: \Gamma \rightarrow I\left(\tilde{M}_{i}\right)$ denote the projection homomorphism for $0 \leq i \leq k$. If $N$ is a nonidentity normal subgroup of $\Gamma$, then for every integer $i \geq 1$ we have either

(1) $p_{i}(N)$ is a discrete subgroup of $I\left(\tilde{M}_{i}\right)$ or

(2) $\tilde{M}_{i}$ is a symmetric space of noncompact type and $I_{0}\left(\tilde{M}_{i}\right) \subseteq \overline{p_{i}(N)}$.

Moreover, if $N$ is discrete, then $p_{i}(\Gamma)$ is discrete for some integer $i \geq 1$ if $p_{i}(N) \neq$ $\{1\}$.

PROOF. We consider first the case that $N$ is an arbitrary nonidentity normal subgroup of $\Gamma$, not necessarily discrete. Suppose that $p_{i}(N)$ is not discrete for

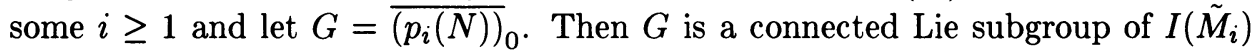
of positive dimension and $G$ is normalized by $p_{i}(\Gamma)$, which satisfies the duality condition by Proposition 1.6b. Since $\tilde{M}_{i}$ is irreducible it follows from Theorem E that $\tilde{M}_{i}$ is a symmetric space of noncompact type and $G=I_{0}\left(\tilde{M}_{i}\right)$.

Now suppose that $N$ is a discrete nonidentity normal subgroup of $\Gamma$ and $p_{i}(N) \neq$ $\{1\}$ for some $i \geq 1$. Suppose that $p_{i}(\Gamma)$ is not discrete and let $G=\left(\overline{p_{i}(\Gamma)}\right)_{0}$. The group $G$ is a connected Lie subgroup of $I\left(\tilde{M}_{i}\right)$ of positive dimension that normalizes $p_{i}(N)$ in $I\left(\tilde{M}_{i}\right)$ and hence normalizes $N$ in $I(\tilde{M})$. By the lemma used to prove assertion (4) of Theorem D it follows that $G$ centralizes $N$ in $I(\tilde{M})$ and hence centralizes $p_{i}(N)$ in $I\left(\tilde{M}_{i}\right)$. This contradicts the corollary to the lemma at the beginning of this section since $\tilde{M}_{i}$ is irreducible. Therefore $p_{i}(\Gamma)$ is discrete.

The next result is Lemma $\mathrm{A}$ of $[\mathbf{E} 1]$.

COROLlary F. Let $\tilde{M}=\tilde{M}_{0} \times \tilde{M}_{1}$, where $\tilde{M}_{0}$ is the Euclidean de Rham factor of $\tilde{M}$ and $\tilde{M}_{1}$ is the product of all non-Euclidean de Rham factors of $\tilde{M}$. Let $p_{i}: I(\tilde{M}) \rightarrow I\left(\tilde{M}_{i}\right)$ denote the projection homomorphism for $i=0,1$. If $\Gamma \subseteq I(\tilde{M})$ is a discrete group that satisfies the duality condition, then $p_{1}\left(\mathrm{I}^{\prime}\right)$ is a discrete subgroup of $I\left(\tilde{M}_{1}\right)$.

ProOF. Let $M=M_{0}^{*} \times M_{1}^{*} \times \cdots \times M_{k}^{*}$ denote the de Rham decomposition of $\tilde{M}$, where $M_{0}^{*}=\tilde{M}_{0}$ and $M_{1}^{*} \times \cdots \times M_{k}^{*}$ is the de Rham decomposition of $\tilde{M}_{1}$. Let $\Gamma^{*}$ denote the subgroup of $\Gamma$ that leaves invariant the de Rham decomposition. Then $\Gamma^{*}$ has finite index in $\Gamma$ since the elements of $I(\tilde{M})$ leave invariant the foliation of $\tilde{M}$ induced by $M_{0}^{*}$ and permute the foliations induced by $M_{i}^{*}$ for $i \geq 1$. Let $p_{i}^{*}: \Gamma^{*} \rightarrow$ $I\left(M_{i}^{*}\right)$ denote the projection homomorphism for $0 \leq i \leq k$. By Proposition $1.6 \mathrm{~b}$ it follows that $\Gamma^{*}$ satisfies the duality condition in $\tilde{M}$ and $p_{i}^{*}\left(\Gamma^{*}\right)$ satisfies the duality condition in $M_{i}^{*}$ for every $i$. Setting $N=\Gamma^{*}$ and applying the previous result to $\Gamma^{*}$ we find that $p_{i}^{*}\left(\Gamma^{*}\right)$ is discrete for every $i \geq 1$. Hence $p_{1}\left(\Gamma^{*}\right)$ is a discrete subgroup of $I\left(\tilde{M}_{1}\right)$ since $p_{1}\left(\Gamma^{*}\right)$ is a subgroup of $p_{1}^{*}\left(\Gamma^{*}\right) \times \cdots \times p_{k}^{*}\left(\Gamma^{*}\right)$. We conclude that $p_{1}(\Gamma)$ is a discrete subgroup of $I\left(\tilde{M}_{1}\right)$ since $p_{1}\left(\Gamma^{*}\right)$ has finite index in $p_{1}(\Gamma)$. 
5. Lattices. Let $\tilde{M}$ be any complete, simply connected manifold with sectional curvature $K \leq 0$.

DEFINITIONS. If $\Gamma \subseteq I(\tilde{M})$ is a discrete subgroup, then $\operatorname{vol}(\tilde{M} / \Gamma)=$ $\inf \{A>0: \operatorname{vol}(O) \leq A$ for any open set $O \subseteq \tilde{M}$ such that $\varphi(O) \cap O$ is empty for all $\varphi \neq 1$ in $\Gamma\}$. A discrete group $\Gamma$ is called a lattice if $\operatorname{vol}(\tilde{M} / \Gamma)$ is finite. A lattice $\Gamma$ is uniform (nonuniform) if the quotient space $\tilde{M} / \Gamma$ is compact (noncompact).

If $\Gamma \subseteq I(\tilde{M})$ is a discrete group, then the set of points in $\tilde{M}$ fixed by some nonidentity element of $\Gamma$ is a closed nowhere dense subset of $\tilde{M}$. If $p \in \tilde{M}$ is a point not fixed by any nonidentity element of $\Gamma$, then we define the Dirichlet fundamental domain $R_{p}$ with center $p$ :

$$
R_{p}=\{q \in \tilde{M}: d(p, q)<d(p, \varphi q) \text { for all } \varphi \neq 1 \text { in } \Gamma\} .
$$

Proposition G. Let $\Gamma \subseteq I(\tilde{M})$ be a discrete group and let $p$ be a point of $\tilde{M}$ that is not fixed by any nonidentity element of $\Gamma$. Then

(1) $R_{p}$ is an open subset of $\tilde{M}$ that contains $p$.

(2) $\varphi\left(R_{p}\right) \cap R_{p}$ is empty for all $\varphi \neq 1$ in $\Gamma$.

(3) $\tilde{M}=\bigcup_{\varphi \in \Gamma} \varphi\left(\overline{R_{p}}\right)$,

(4) $\operatorname{vol}\left(R_{p}\right)=\operatorname{vol}(\tilde{M} / \Gamma)$.

We omit the proof, which is routine.

Proposition H. Let $\Gamma \subseteq I(\tilde{M})$ be a lattice, and let $\tilde{M}=\tilde{M}_{1} \times \tilde{M}_{2}$ be a Riemannian splitting that is invariant under $\Gamma$. Let $p_{i}: \Gamma \rightarrow I\left(\tilde{M}_{i}\right)$ be the projection homomorphism for $i=1,2$. If $p_{2}(\Gamma)$ is a discrete subgroup of $I\left(\tilde{M}_{2}\right)$, then $p_{2}(\Gamma)$ is a lattice in $I\left(\tilde{M}_{2}\right)$ and $\operatorname{kernel}\left(p_{2}\right)$ is a lattice in $I\left(\tilde{M}_{1}\right)$.

REMARK. This result not only strengthens the conclusion of assertion (1) in Theorem 4.1 of [E5] but it also considerably simplifies the proof.

PROOF. Let $N$ denote the kernel of $p_{2}$. Clearly $N$ is a discrete subgroup of $I\left(\tilde{M}_{1}\right)$. Let $O_{1} \subseteq \tilde{M}_{1}$ be any open set such that $\varphi\left(O_{1}\right) \cap O_{1}$ is empty for all $\varphi \neq 1$ in $N$. Let $O_{2} \subseteq \tilde{M}_{2}$ be any open set such that $\varphi_{2}\left(O_{2}\right) \cap O_{2}$ is empty for all $\varphi_{2} \neq 1$ in $p_{2}(\Gamma)$; such open sets exist by the discreteness of $p_{2}(\Gamma)$. If $O=O_{1} \times O_{2}$, then $O$ is open in $\tilde{M}=\tilde{M}_{1} \times \tilde{M}_{2}$ and it follows from the definition of $O_{1}$ and $O_{2}$ that $\varphi(O) \cap O$ is empty for all $\varphi \neq 1$ in $\Gamma$. Since $\Gamma \subseteq I(\tilde{M})$ is a lattice there exists a number $A>0$ depending only on $\Gamma$ such that $\operatorname{vol}\left(O_{1}\right) \cdot \operatorname{vol}\left(O_{2}\right)=\operatorname{vol}(O) \leq A$. Hence $\operatorname{vol}\left(O_{1}\right) \leq A / \operatorname{vol}\left(O_{2}\right)$. By the arbitrary choice of the open set $O_{1}$ it follows that $N=\operatorname{kernel}\left(p_{2}\right)$ is a lattice in $I\left(\tilde{M}_{1}\right)$. Similarly $\operatorname{vol}\left(O_{2}\right) \leq A / \operatorname{vol}\left(O_{1}\right)$ and by the arbitrary choice of the open set $O_{2}$ it follows that $p_{2}(\Gamma)$ is a lattice in $I\left(\tilde{M}_{2}\right)$.

The next result is a restatement of the main theorem of [E1], but the proof here simplifies greatly the proof of $[\mathbf{E} 1]$. The next result is also a special case of Proposition 2.4 and Theorem 4 of [E4], but the extra generality in [E4] at present requires a much more complicated proof.

THEOREM I. Let $\tilde{M}=\tilde{M}_{0} \times \tilde{M}_{1}$, where $\tilde{M}_{0}$ is the Euclidean de Rham factor of $\tilde{M}$ and $\tilde{M}_{1}$ is the product of all non-Euclidean de Rham factors of $\tilde{M}$. Let 
$p_{i}: I(\tilde{M}) \rightarrow I\left(\tilde{M}_{i}\right)$ denote the projection homomorphism for $i=0,1$. Let $\Gamma \subseteq I(\tilde{M})$ be a lattice. Then

(1) $\operatorname{kernel}\left(p_{1}\right)$ is a uniform lattice in $\tilde{M}_{0}$.

(2) The dimension of $\tilde{M}_{0}$ equals the rank of the Clifford subgroup of $\Gamma$, the unique maximal normal abelian subgroup of $\Gamma$.

PROOF. By Corollary $\mathrm{F}$ of the previous section the group $p_{1}(\Gamma)$ is discrete in $I\left(\tilde{M}_{1}\right)$, and by Proposition $\mathrm{H}$ above we conclude that $\operatorname{kernel}\left(p_{1}\right)$ is a lattice in $\tilde{M}_{0}$. A theorem of Bieberbach [W2, p. 100] says that $C_{1}=\operatorname{kernel}\left(p_{1}\right) \cap C\left(\tilde{M}_{0}\right)$ is a normal abelian subgroup of finite index in $\operatorname{kernel}\left(p_{1}\right)$. Hence both $C_{1}$ and $\operatorname{kernel}\left(p_{1}\right)$ are uniform lattices in $I\left(\tilde{M}_{0}\right)$, which proves (1).

If $C(\Gamma)=\Gamma \cap C(\tilde{M})$, then $C(\Gamma)$ is a normal abelian subgroup of $\Gamma$ by the discussion in (1.4), and $C(\Gamma)$ is the unique maximal abelian normal subgroup of $\Gamma$ by Theorem B of $\S 4$. The group $C_{1} \subseteq C\left(\tilde{M}_{0}\right)$ defined above is a uniform lattice in $I\left(\tilde{M}_{0}\right)$, and hence $\operatorname{dim} \tilde{M}_{0}=\operatorname{rank} C_{1} \leq \operatorname{rank} C(\Gamma)$ since $C_{1} \subseteq C(\Gamma)$. Conversely, $C(\Gamma)$ is a discrete subgroup of $C\left(\tilde{M}_{0}\right) \times\{1\}$, and hence $\operatorname{rank} C(\Gamma) \leq \operatorname{dim} \tilde{M}_{0}$, which completes the proof of (2) and of the theorem.

We conclude with two results on lattices that are nontrivial direct products of subgroups. The first of these results is Theorem 2 of $[\mathbf{S}]$. It was proved in the compact $C^{\infty}$ case by Gromoll and Wolf $[\mathbf{G W}]$ and in the compact real analytic case by Lawson and Yau [LY]. The second result is similar to Proposition 2.6 of [E4].

THEOREM J. Let $\Gamma \subseteq I(\tilde{M})$ be a lattice with trivial center that splits as a direct product $\Gamma_{1} \times \Gamma_{2}$ of subgroups $\Gamma_{1}$ and $\Gamma_{2}$. Then there exists a Riemannian splitting $\tilde{M}=\tilde{M}_{1} \times \tilde{M}_{2}$ that is invariant under $\Gamma$ and such that $\Gamma_{1} \subseteq I\left(\tilde{M}_{1}\right) \times\{1\}$ and $\Gamma_{2} \subseteq\{1\} \times I\left(\tilde{M}_{2}\right)$. In particular $\Gamma_{i}$ is a lattice in $I\left(\tilde{M}_{i}\right)$ for $i=1,2$.

Proof. Let $\tilde{M}=\tilde{M}_{0} \times \tilde{M}_{1} \times \tilde{M}_{2}$ be the $\Gamma$-invariant splitting of $\tilde{M}$ that has the properties of the statement of Theorem D of $\S 4$, where we set $A=\Gamma_{1}$ and $B=\Gamma_{2}$ in the notation of that result. By Theorem D it suffices to show that $\tilde{M}_{0}$ has dimension zero. Suppose that $\tilde{M}_{0}$ has positive dimension and let $M_{1}^{*}$ denote $\tilde{M}_{1} \times \tilde{M}_{2}$, with $p_{1}^{*}: \Gamma \rightarrow I\left(M_{1}^{*}\right)$ denoting the corresponding projection homomorphism. By assertion (4) of Theorem D the group $p_{1}(\Gamma) \times p_{2}(\Gamma)$ is discrete in $I\left(M_{1}^{*}\right)$ and hence the subgroup $p_{1}^{*}(\Gamma)$ is also discrete. By Proposition $\mathrm{H}$ we see that $\operatorname{kernel}\left(p_{1}^{*}\right)$ is a lattice in $I\left(\tilde{M}_{0}\right)$ and in particular is not the identity. By assertion (1) of Theorem D we see that $\operatorname{kernel}\left(p_{1}^{*}\right) \subseteq C\left(\tilde{M}_{0}\right)$ and hence $\operatorname{kernel}\left(p_{1}^{*}\right)$ lies in the center of $\Gamma$ by assertion (2) of Theorem D. This contradicts the hypothesis that $\Gamma$ has trivial center and completes the proof of Theorem $\mathbf{J}$.

THEOREM K. Let $\Gamma \subseteq I(\tilde{M})$ be a lattice that splits as a direct product $\Gamma_{1} \times \Gamma_{2}$ of subgroups $\Gamma_{1}$ and $\Gamma_{2}$. Then for $i=1,2$ the group $\Gamma_{i}$ is isomorphic to a lattice in a Riemannian factor of $\tilde{M}$.

REMARK. It is not necessarily true that each group $\Gamma_{i}$ is itself a lattice in some Riemannian factor. See for example the proof of Proposition 3.2 of [E4].

PROOF OF THE THEOREM. For later convenience of notation we set $A=\Gamma_{1}$ and $B=\Gamma_{2}$ so that $\Gamma$ is the direct product $A \cdot B$. Let $\tilde{M}=\tilde{M}_{0} \times \tilde{M}_{1} \times \tilde{M}_{2}$ be the $\Gamma$ invariant splitting of Theorem $\mathrm{D}$ in the previous section. We may assume that $\tilde{M}_{0}$ 
has positive dimension for otherwise we are done by Theorem D. Let $A_{0}, B_{0}$ and $\Gamma_{0}$ denote respectively the centers of $A, B$ and $\Gamma$. In particular $A_{0} \cap B_{0}=\{1\}$ and $\Gamma_{0}$ is the direct product $A_{0} \cdot B_{0}$. By (4) of Theorem D the projection $\left(p_{1} \times p_{2}\right)(\Gamma)$ is discrete in $I\left(\tilde{M}_{1} \times \tilde{M}_{2}\right)$ and it now follows from (1) of Theorem D and Proposition $\mathrm{H}$ that $\Gamma_{0}=\operatorname{kernel}\left(p_{1} \times p_{2}\right)$ is a uniform lattice in $I\left(\tilde{M}_{0}\right)$.

It suffices to prove the theorem for the subgroup $A$. We prove the result in three steps.

Step $1 . \Gamma^{*}=\operatorname{kernel}\left(p_{2}\right)$ is a lattice in $I\left(\tilde{M}_{0} \times \tilde{M}_{1}\right)$ and $\Gamma^{*}=A \cdot B_{0}$.

ProOF. By (4) of Theorem D and Proposition $\mathrm{H}$ it follows that $\Gamma^{*}=\operatorname{kernel}\left(p_{2}\right)$ is a lattice in $I\left(\tilde{M}_{0} \times \tilde{M}_{1}\right)$. Clearly $A \cdot B_{0} \subseteq \Gamma^{*}$ by assertion (3) of Theorem D and the fact that $B_{0} \subseteq \Gamma_{0}=\operatorname{kernel}\left(p_{1} \times p_{2}\right)$. Conversely let $\varphi \in \Gamma^{*} \subseteq \Gamma$ be given and write $\varphi=a b$ for suitable elements $a \in A$ and $b \in B$. Then $b=a^{-1} \varphi \in \Gamma^{*}$ by assertion (3a) of Theorem $\mathrm{D}$, and it follows that $b \in C\left(\tilde{M}_{0}\right)$ by assertion (3b) of Theorem D. Therefore $b \in B_{0}$ and $\varphi \in A \cdot B_{0}$ by assertion (2) of Theorem D. This completes the proof of Step 1.

Before stating Steps 2 and 3 we need some preliminary discussion. We may assume that $B_{0} \neq\{1\}$ for otherwise $\Gamma^{*}=A$ is a lattice in $I\left(\tilde{M}_{0} \times \tilde{M}_{1}\right)$ by Step 1. By assertion (1) of Theorem $\mathrm{D}$ the group $B_{0}$ (and in fact $p_{0}(\Gamma)$ ) consists of translations of the Euclidean space $\tilde{M}_{0}$. Hence we may write

$$
\tilde{M}_{0}=\tilde{M}_{\alpha} \times \tilde{M}_{\beta}, \quad \text { Riemannian product }
$$

such that $B_{0} \subseteq\{1\} \times I\left(\tilde{M}_{\beta}\right)$ and $B_{0}$ is a uniform lattice of $I\left(\tilde{M}_{\beta}\right)$. This splitting of $\tilde{M}_{0}$ is invariant under the abelian group $p_{0}(\Gamma)$ since $B_{0}$ is a normal subgroup of $p_{0}(\Gamma)$. Hence the splitting $\tilde{M}_{0} \times \tilde{M}_{1}=\tilde{M}_{\alpha} \times \tilde{M}_{\beta} \times \tilde{M}_{1}$ of $\tilde{M}_{0} \times \tilde{M}_{1}$ is invariant under $\Gamma^{*}=\operatorname{kernel}\left(p_{2}\right)$. Let $p_{\alpha}, p_{\beta}$ and $p_{1}$ denote the projection homomorphisms of $\Gamma^{*}$ into $I\left(\tilde{M}_{\alpha}\right), I\left(\tilde{M}_{\beta}\right)$ and $I\left(\tilde{M}_{1}\right)$ respectively.

Step 2. $p_{\alpha} \times p_{1}: A \rightarrow\left(p_{\alpha} \times p_{1}\right)(A)=\left(p_{\alpha} \times p_{1}\right)\left(\Gamma^{*}\right)$ is an isomorphism.

Step 3. $\left(p_{\alpha} \times p_{1}\right)\left(\Gamma^{*}\right)$ is discrete in $I\left(\tilde{M}_{\alpha} \times \tilde{M}_{1}\right)$.

These two steps will complete the proof of the theorem since $\left(p_{\alpha} \times p_{1}\right)\left(\Gamma^{*}\right)$ will then be a lattice in $I\left(\tilde{M}_{\alpha} \times \tilde{M}_{1}\right)$ by Proposition $\mathrm{H}$ together with the fact that $\Gamma^{*}$ is a lattice in $I\left(\tilde{M}_{0} \times \tilde{M}_{1}\right)$.

PROOF OF STEP 2. Observe that $\left(p_{\alpha} \times p_{1}\right)(A)=\left(p_{\alpha} \times p_{1}\right)\left(\Gamma^{*}\right)$ by the definition of $\tilde{M}_{\beta}$ and the fact that $\Gamma^{*}=A \cdot B_{0}$. To complete the proof of Step 2 it suffices to show that $A \cap \operatorname{kernel}\left(p_{\alpha} \times p_{1}\right)=\{1\}$. Let $\varphi \in A$ be an element such that $p_{\alpha}(\varphi)=$ $p_{1}(\varphi)=\{1\}$. By assertion (3) of Theorem D it follows that $\varphi=\left(\mathrm{id}, \varphi_{\beta}\right.$, id) $\in$ $I\left(\tilde{M}_{\alpha} \times \tilde{M}_{\beta} \times \tilde{M}_{1}\right)$, where $\varphi_{\beta}$ is a translation on $\tilde{M}_{\beta}$. The group $\Gamma^{\prime}$ generated by $\varphi$ and $B_{0}$ is a discrete subgroup of translations of $\tilde{M}_{\beta}$ since $\Gamma^{\prime} \subseteq \Gamma$ and $\Gamma$ is discrete. Since $B_{0}$ is a uniform lattice in $I\left(\tilde{M}_{\beta}\right)$ it is possible for $\Gamma^{\prime}$ to be discrete only if $B_{0}$ has finite index in $\Gamma^{\prime}$, which means that $\varphi^{n} \in B_{0}$ for some positive integer $n$. Since $\varphi^{n} \in A \cap B_{0}=\{1\}$ and $\varphi$ is a translation of $\tilde{M}_{\beta}$ it follows that $\varphi=\{1\}$.

PROOF OF STEP 3 . Let $\left\{\varphi_{n}\right\} \subseteq A$ be a sequence such that $\left(p_{\alpha} \times p_{1}\right)\left(\varphi_{n}\right) \rightarrow 1$ as $n \rightarrow+\infty$. To prove that $\left(p_{\alpha} \times p_{1}\right)\left(\Gamma^{*}\right)=\left(p_{\alpha} \times p_{1}\right)(A)$ is discrete in $I\left(\tilde{M}_{\alpha} \times \tilde{M}_{1}\right)$ it suffices to show that $p_{\alpha}\left(\varphi_{n}\right)=p_{1}\left(\varphi_{n}\right)=\{1\}$ for sufficiently large $n$. By hypothesis $p_{1}\left(\varphi_{n}\right) \rightarrow 1$ as $n \rightarrow \infty$ and hence $p_{1}\left(\varphi_{n}\right)=\{1\}$ for sufficiently large $n$ since $p_{1}(\Gamma)$ 
is discrete in $I\left(\tilde{M}_{1}\right)$ by assertion (4) of Theorem D. Since $B_{0}$ is a uniform lattice in $I\left(\tilde{M}_{\beta}\right)$ we may choose a sequence $\left\{\beta_{n}\right\} \subseteq B_{0}$ so that $p_{\beta}\left(\varphi_{n} \beta_{n}\right)$ converges to some element $\beta^{*}$ of $I\left(\tilde{M}_{\beta}\right)$ as $n \rightarrow \infty$. The elements $\psi_{n}=\varphi_{n} \beta_{n}$ lie in $\Gamma^{*}=A \cdot B_{0}$ and since $B_{0} \subseteq \operatorname{kernel}\left(p_{\alpha}\right) \cap \operatorname{kernel}\left(p_{1}\right)$ we see that

$$
\begin{aligned}
& p_{\alpha}\left(\psi_{n}\right)=p_{\alpha}\left(\varphi_{n}\right) \rightarrow 1 \quad \text { as } n \rightarrow+\infty, \\
& p_{\beta}\left(\psi_{n}\right) \rightarrow \beta^{*} \quad \text { as } n \rightarrow+\infty, \\
& p_{1}\left(\psi_{n}\right)=p_{1}\left(\varphi_{n}\right)=1 \quad \text { for large } n .
\end{aligned}
$$

Hence $\psi_{n} \rightarrow\left(\mathrm{id}, \beta^{*}\right.$,id) as $n \rightarrow \infty$. Since $\Gamma^{*}$ is discrete we conclude that $\psi_{n}=\beta^{*}$ and $p_{\alpha}\left(\psi_{n}\right)=1$ for sufficiently large $n$. This completes the proof of Step 3 and of the theorem.

\section{REFERENCES}

[B] W. Ballmann, Nonpositively curved manifolds of higher rank, Ann. of Math. (2) 122 (1985), $597-609$.

[BBE] W. Ballmann, M. Brin and P. Eberlein, Structure of manifolds of nonpositive curvature, I, Ann. of Math. (2) 122 (1985), 171-203.

[BGS] W. Ballmann, M. Gromov and V. Schroeder, Manifolds of nonpositive curvature, Birkhäuser, 1985.

[BO] R. Bishop and B. O'Neill, Manifolds of negative curvature, Trans. Amer. Math. Soc. 145 (1969), 1-49.

[BS] K. Burns and R. Spatzier, Manifolds of nonpositive curvature and their buildings, Publ. Inst. Hautes Etudes Sci. 65 (1987), 35-59.

[CE] S. Chen and P. Eberlein, Isometry groups of simply connected manifolds of nonpositive curvature, Illinois J. Math. 24 (1980), 73-103.

[E1] P. Eberlein, Euclidean de Rham factor of a lattice of nonpositive curvature, J. Differential Geometry 18 (1983), 209-220.

[E2] _ Geodesic flows on negatively curved manifolds, I, Ann. of Math. (2) 95 (1972), 492-510.

[E3] _ Isometry groups of simply connected manifolds of nonpositive curvature, II, Acta Math. 149 (1982), 41-69.

[E4] __ L-subgroups in spaces of nonpositive curvature, Curvature and Topology of Riemannian Manifolds, Proc. Katata, 1985, Lecture Notes in Math., vol. 1201, Springer, Berlin, 1986, pp. 41-88.

[E5] _ Lattices in spaces of nonpositive curvature, Ann. of Math. (2) 111 (1980), 435-476.

[E6] _ Rigidity of lattices of nonpositive curvature, Ergodic Theory Dynamical Systems 3 (1983), 47-85.

[E7] _ Structure of manifolds of nonpositive curvature, Global Differential Geometry and Global Analysis, 1984, Lecture Notes in Math., vol. 1156, Springer, Berlin, 1985, pp. 86-153.

[E8] _ Symmetry diffeomorphism group of a manifold of nonpositive curvature, II (submitted).

[EO] P. Eberlein and B. O'Neill, Visibility manifolds, Pacific J. Math. 46 (1973), 45-109.

[GW] D. Gromoll and J. Wolf, Some relations between the metric structure and the algebraic structure of the fundamental group in manifolds of nonpositive curvature, Bull. Amer. Math. Soc. 77 (1971), 545-552.

[H] S. Helgason, Differential geometry and symmetric spaces, Academic Press, New York, 1962.

[K] F. I. Karpelevic, The geometry of geodesics and the eigenfunctions of the Beltrami-Laplace operator on symmetric spaces, Trans. Moscow Math. Soc. 14 (1965), 51-199.

[KN] S. Kobayashi and K. Nomizu, Foundations of differential geometry, vol. 1, Wiley, New York, 1963, pp. 179-193.

[LY] H. B. Lawson and S.-T. Yau, Compact manifolds of nonpositive curvature, J. Differential Geometry 7 (1972), 211-228.

[L] O. Loos, Symmetric spaces, vol. 1, Benjamin, New York, 1969.

[M] G. D. Mostow, Strong rigidity of locally symmetric spaces, Ann. of Math. Studies, No. 78, Princeton Univ. Press, Princeton, N.J., 1973. 
[S] V. Schroeder, A splitting theorem for spaces of nonpositive curvature, Invent. Math. 79 (1985), 323-327.

[W1] J. Wolf, Homogeneity and bounded isometries in manifolds of negative curvature, Illinois $\mathrm{J}$. Math. 8 (1964), 14-18.

[W2] _ Space of constant curvature, 2nd ed., published by the author, Berkeley, 1972.

Department of Mathematics, University of North Carolina, Chapel Hill, NORTH CAROLINA 27514

Current address (August 1988-1989): Department of Mathematics, University of Pennsylvania, Philadelphia, Pennsylvania 19104 